\title{
Vergleich der Antibiotika-Empfindlichkeitsbestimmung mit dem COBAS-Bact-System und der Bouillon-Dilutionsmethode (DIN 58940)
}

\author{
K.-H. Buscher, S. Binder, U. Deiß, M. Stieglitz \\ Abteilung fur Medizinische Mikrobiologie und Immunologie, Ruhr-Universität Bochum
}

\begin{abstract}
Zusammenfassung:
Es wurden 421 klinische Isolate verschiedener Bakterienstämme in Hinblick auf ihre Empfindlichkeit gegenüber 15 verschiedenen Antibiotika mit Hilfe des COBAS-Bact und der Bouillon-Dilution (DIN 58940) untersucht. Es zeigte sich eine totale Übereinstimmung von $71,1 \%$ und eine totale Übereinstimmung plus kleinere Abweichungen von 90,7\%. Sehr große Abweichungen betrugen bis zu 97\% (Streptococcus faecalis/ Norfloxacin) in Abhängigkeit vom Antibiotikum und der Keimspezies. Die Untersuchung der Tag-zu-TagReproduzierbarkeit ergab in einigen Fällen auch Abweichungen um zwei Beurteilungsstufen.
\end{abstract}

Insgesamt bedarf das Gerät hinsichtlich der Präzision und Richtigkeit der Antibiotika-Empfindlichkeitsbestimmung sicherlich noch weiterer Verbesserungen.

Schlüsselwörter:

Empfindlichkeitsprüfung von Bakterien - Automatisierung in der.Mikrobiologie - Bouillon-Verdünnungstest - Minimale Hemmkonzentration

\begin{abstract}
Summary:
421 clinical isolates of different bacterial species were studied for their susceptibility against 15 different antibiotics by use of either the COBAS Bact system or the broth dilution method (according to DIN 58940). Total agreement was found in $71.1 \%$ and total agreement plus minor discrepancies in $90.7 \%$ of the bacteria/ antibiotic combinations tested. Very major errors could be found up to $97 \%$ (Streptococcus faecalis/ norfloxacin) depending on the antibiotic and bacterial species tested. Studies concerning the day to day precision showed in some cases major discrepancies.
\end{abstract}

It seems that this instrument needs further improvement in regard to precision and validity of antibiotic susceptibility testing.

Keywords:

susceptibility testing of bacteria - automation in microbiology - broth dilution test - minimal inhibitory concentration

\section{Einleitung}

In den letzten Jahren zeigte sich auch im Bereich der. klinischen Mikrobiologie eine zunehmende Tendenz zur Entwicklung automatisierter Verfahren zur Identifikation und Antibiotika-Empfindlichkeitsbestimmung von Bakterien (5, 11). Ein Gerät, welches dieser Entwicklung folgt und sowohl eine Identifikation von Enterobacteriaceae als auch eine Empfindlichkeitsbestimmung von schnellwachsenden Mikroorganismen innerhalb von $5 \mathrm{Std}$. erlaubt, ist der COBAS-Bact (Hoffmann La Roche, Basel, Schweiz) $(2,12)$. Darüber hinaus ist dieses Gerät durch frei programmierbare Forschungsprogramme und Analyse der turbidimetrisch erstellten Wachstumskurven für eine vielseitige Anwendung auch in der Mutagenitätsanalyse geeignet $(1,8)$. Vielfache Untersuchungen zur Übereinstimmung der Ergebnisse der Empfindlichkeitsprüfung mit dem COBAS-Bact mit solchen von Agardiffusions- oder Agardilutionsmethoden nach DIN oder
NCCLS zeigen zumeist Übereinstimmungen von $90 \%$ und höher $(4,6,10)$. Aus diesen Studien ist nicht ersichtlich, wie das Resistenzverhalten der untersuchten Bakterien in Hinblick auf die Standardmethode war. Das Ergebnis eines Methodenvergleiches ist aber abhängig von den verwendeten Testkeimen. Haben diese eine minimale Hemmkonzentration (MHK), die entweder im sensitiven oder resistenten Bereich weit vom „breakpoint" entfernt liegen, ist auf jeden Fall mit einer hohen Übereinstimmungsrate zu rechnen. Außerdem liegen zur Zeit keine vergleichbaren Untersuchungen mit Bestimmungen der minimalen Hemmkonzentration vor. Aus diesem Grunde wurden in der vorliegenden Studie 421 verschiedene klinische Isolate unterschiedlicher Bakterienstämme mit MHK's ausgewählt, die möglichst nahe am „breakpoint" lagen. Damit konnten geringe Diskrepanzen der Bestimmung schon zu einer unterschiedlichen Bewertung im Sinne von sensibel, intermediär sensitiv oder resistent führen. Die Vergleichsuntersuchungen wurden mit der Mikrodilutionsmethode nach DIN 58940 durchgeführt. . 


\section{Material und Methoden}

\section{Antibiotika}

Antibiotika-Testsubstanzen wurden uns freundlicherweise von folgenden Firmen zur Verfügung gestellt: Ampicillin, Penicillin und Oxacillin von Bayer AG, Leverkusen; Piperacillin von Cyanamid Lederle, Wolfratshausen; Ceftazidim von Cascan, Wiesbaden; Ceftriaxon und Cotrimoxazol von Hoffmann La Roche, Basel; Gentamicin von Merck, Darmstadt; Amoxycillin/Clavulansäure von Beecham-Wülfing, Neuss; Cefuroxim von Hoechst AG, Frankfurt; Latamoxef und Cefazolin von Eli Lilly, Bad Homburg; Norfloxacin von Merck, Sharp und Dohme, München; Cefoperazon von Pfizer, Karlsruhe; Cefsulodin von Takeda-Grünenthal, Stolberg.

Antibiotika-Testblättchen für den COBAS-Bact wurden uns freundlicherweise von Hoffmann La Roche, Grenzach, zur Verfügung gestellt.

\section{Bakterien}

Es wurden verschiedene Stämme unterschiedlicher Bakterienarten, die aus klinischem Untersuchungsmaterial isoliert wurden, untersucht. Alle gram-negativen Keime wurden mit dem API 20E oder dem API 20NE System (API Bio Merieux, Nürtingen) identifiziert. Die Identifizierung der gram-positiven Keime erfolgte nach Standardmethoden (7). Tab. 1 zeigt die genaue Anzahl der jeweils untersuchten Stämme. Die Antibiotika-Empfindlichkeitseigenschaften der untersuchten Gesamtpopulation sind in Tab. 2 dargestellt.

\section{Empfindlichkeitsprüfung}

a) Bestimmung der minimalen Hemmkonzentration

Die minimale Hemmkonzentration wurde durch zweifach serielle Verdünnung der Antibiotika in Mueller-HintonBouillon (Merck, Darmstadt) bestimmt. Das Gesamtvolumen betrug $100 \mu \mathrm{l}$ und das Inokulum $5 \times 10^{-5} \mathrm{Keime} / \mathrm{ml}$ (DIN 58940). Die Beurteilung erfolgte nach einer Inkubationszeit von 18 Std. nach den in DIN 58940 (3) festgelegten Grenzwerten.

Tab. 1: Anzahl der untersuchten verschiedenen Bakterienstämme

$\overline{\text { Bakterien }}$ n

Escherichia coli

Klebsiella spec.

Serratia spec.

Enterobacter cloacae

Citrobacter freundii

Morganella morganii

Proteus mirabilis

Proteus vulgaris

Providencia rettgeri

Pseudomonas aeruginosa

Acinetobacter calcoaceticus

Yersinia spec.

Staphylococcus aureus

Streptococcus faecalis

Streptococcus pyogenes

Streptococcus agalactiae b) COBAS-Bact

Die Empfindlichkeitsprüfung mit dem COBAS-Bact wurde entsprechend den Anweisungen des Herstellers durchgeführt. In Kürze: Bakterien wurden nach Übernachtkultur von Blutagarplatten abgeimpft, in $2 \mathrm{ml} \mathrm{CO}$. BAS-Bact-Bouillon suspendiert, auf eine Trübung von $0,5 \mathrm{McFarland}$-Einheiten eingestellt und $100 \mu \mathrm{l}$ (gramnegative Keime), bzw. $200 \mu$ l (gram-positive Keime) ins Zentrum des Meßrotors pipettiert. Anschließend wurden $5 \mathrm{ml}$ COBAS-Bact-Bouillon zugegeben und der verschlossene Rotor in das System geladen. Nach 5 Std. erfolgte die Ausgabe der Beurteilung.

Der Vergleich der beiden Methoden erfolgte nach folgendem Schema:

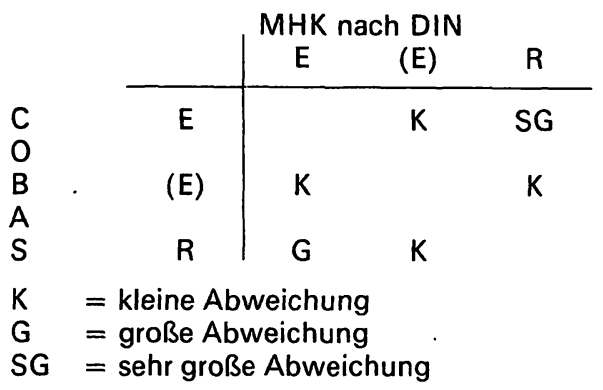

\section{Ergebnisse}

Eine Empfindlichkeitsprüfung mit dem COBAS-Bact-System ist nicht für alle Bakterienarten durchführbar. So ließen sich Streptococcus pyogenes gar nicht und Acinetobacter calcoaceticus und Yersinia spec. nur zu 17\%, bzw. zu $24 \%$ testen. In diesen Fällen druckt das Gerät "growth control negative" als Ergebnis aus.

Die Ergebnisse der Empfindlichkeitsprüfung verschiedener Bakterienarten mit dem COBAS-Bact im Vergleich zur Bouillon-Dilutions-Methode sind in Tab. 3 dargestellt. Bei der Gesamtauswertung zeigt sich, daß die großen Abweichungen mit insgesamt $2,0 \%$ in einem vertretbaren Rahmen liegen, dagegen sind die sehr großen Abweichungen mit 7,3\% gefährlich hoch. Diese Keime sind nach dem Reihenverdünnungstest resistent, werden aber mit

Tab.2: Antibiotika-Empfindlichkeitsverteilung in der Gesamtpopulation (Bouillon-Dilution; $n=$ Anzahl der untersuchten Stämme)

\begin{tabular}{lrrrr}
\hline Antibiotikum & $\mathbf{n}$ & \multicolumn{2}{c}{$\%$ Anteil } \\
\cline { 3 - 5 } & & $\begin{array}{c}\text { sen- } \\
\text { sitiv }\end{array}$ & $\begin{array}{c}\text { intermediär } \\
\text { sensitiv }\end{array}$ & $\begin{array}{c}\text { re- } \\
\text { sistent }\end{array}$ \\
\hline Ampicillin & 341 & 16,7 & 1,8 & 81,5 \\
Piperacillin & 363 & 33,6 & 30,0 & 36,4 \\
Ceftazidim & 283 & 85,9 & 10,2 & 3,9 \\
Ceftriaxon & 282 & 74,8 & 8,5 & 16,7 \\
Gentamicin & 367 & 46,3 & 37,1 & 16,6 \\
Cotrimoxazol & 338 & 75,7 & 2,1 & 22,2 \\
Amoxy/Clav. & 320 & 33,4 & 5,3 & 61,3 \\
Cefuroxim & 344 & 15,7 & 39,8 & 44,5 \\
Latamoxef & 274 & 83,9 & 9,5 & 6,6 \\
Cefazolin & 339 & 21,2 & 12,1 & 66,7 \\
Norfloxacin & 368 & 70,9 & 0 & 29,1 \\
Cefoperazon & 278 & 49,6 & 23,0 & 27,4 \\
Cefsulodin & 26 & 15,4 & 80,7 & 3,9 \\
Penicillin & 49 & 32,7 & 18,4 & 48,9 \\
Oxacillin & 69 & 37,7 & 0 & 62,3 \\
\hline
\end{tabular}


Tab. 3: Abweichungen der Ergebnisse der Empfindlichkeitsprü. fung verschiedener Bakterienarten mit Hilfe des COBAS-Bact im Vergleich zur Mikrodilutionsmethode (DIN 58940). ( $n=$ Gesamtzahl der Untersuchungen, d.h. Zahl der untersuchten Stämme x Anzahl der getesteten Antibiotika)

\begin{tabular}{|c|c|c|c|c|}
\hline \multirow[t]{2}{*}{ Bakterien } & \multirow[t]{2}{*}{$\mathbf{n}$} & \multicolumn{3}{|c|}{ Abweichungen in \% } \\
\hline & & kleine & große & sehr große \\
\hline $\begin{array}{l}\text { Escherichia coli } \\
\text { Klebsiella spec. } \\
\text { Serratia spec. } \\
\text { Enterobacter } \\
\text { cloacae }\end{array}$ & $\begin{array}{l}571 \\
348 \\
348 \\
360\end{array}$ & $\begin{array}{l}25,0 \\
19,3 \\
17,2 \\
18,1\end{array}$ & $\begin{array}{l}7,9 \\
0,9 \\
3,4 \\
0,0\end{array}$ & $\begin{array}{l}1,8 \\
6,0 \\
5,7 \\
5,6\end{array}$ \\
\hline $\begin{array}{l}\text { Citrobacter } \\
\text { freundii }\end{array}$ & 360 & 15,5 & 1.1 & 8,1 \\
\hline $\begin{array}{l}\text { Proteus mirabilis } \\
\text { Providencia } \\
\text { rettgeri }\end{array}$ & $\begin{array}{r}203 \\
96\end{array}$ & $\begin{array}{l}16,7 \\
15,6\end{array}$ & $\begin{array}{l}0,0 \\
2,1\end{array}$ & $\begin{array}{l}5,9 \\
7,3\end{array}$ \\
\hline $\begin{array}{l}\text { Proteus vulgaris } \\
\text { Morganella } \\
\text { morganii }\end{array}$ & $\begin{array}{l}358 \\
320\end{array}$ & $\begin{array}{r}8.4 \\
11,9\end{array}$ & $\begin{array}{l}0,0 \\
1,3\end{array}$ & $\begin{array}{l}9,2 \\
5,6\end{array}$ \\
\hline $\begin{array}{l}\text { Yersinia spec. } \\
\text { Acinetobacter } \\
\text { calcoaceticus }\end{array}$ & $\begin{array}{l}66 \\
39\end{array}$ & $\begin{array}{l}25,8 \\
23,1\end{array}$ & $\begin{array}{r}15,2 \\
0,0\end{array}$ & $\begin{array}{l}0,0 \\
0,0\end{array}$ \\
\hline $\begin{array}{l}\text { Pseudomonas } \\
\text { aeruginosa }\end{array}$ & 201 & 46,8 & 0,5 & 8,5 \\
\hline $\begin{array}{l}\text { Staphylococcus } \\
\text { aureus }\end{array}$ & 305 & 16,1 & 0,7 & 15,4 \\
\hline $\begin{array}{l}\text { Streptococcus } \\
\text { faecalis }\end{array}$ & 319 & 28,2 & 0,3 & 13,8 \\
\hline $\begin{array}{l}\text { Streptococcus } \\
\text { agalactiae }\end{array}$ & 135 & 11,1 & 0,7 & 10,4 \\
\hline Gesamt & 4008 & 19,6 & 2,0 & 7,3 \\
\hline
\end{tabular}

dem COBAS-Bact als sensibel bestimmt. Die kleinen Abweichungen sind mit $19,6 \%$ ebenfalls sehr hoch, was bedeutet, daß nur in $71,1 \%$ eine totale Übereinstimmung gefunden wird. Bei Betrachtung der Ergebnisse für einzelne Keimgruppen fällt die hohe Variationsbreite auf, die z. B. bei den sehr großen Abweichungen von 0,0 bis $15,4 \%$ geht, wobei allerdings kein gehäuftes Auftreten hoher Abweichungen in allen Abweichungsklassen bei bestimmten Bakterienarten beobachtet werden konnte.

Betrachtet man die Ergebnisse bezogen auf einzelne Antibiotika (Tab.4), so zeigt sich, daß insbesondere bei Cefsulodin, Cefuroxim, Gentamicin und Piperacillin häufig kleine Abweichungen in der Beurteilung der Empfindlichkeit auftreten, während bei Ceftazidim verhältnismäßig häufig große und bei Ampicillin, Norfloxacin und Cefoperazon sehr große Abweichungen gehäuft auftreten (Tab.4). Die hohe Zahl sehr großer Abweichungen bei Norfloxacin ist dabei auf die schlechte Korrelation der Ergebnisse beider Methoden vor allem bei gram-positiven Bakterien zurückzuführen (Tab.5).

Die Einzeldarstellung einiger Keim-Antibiotikum-Kombinationen, bei denen entweder die großen oder die sehr großen Abweichungen größer sind als 1.5\% (Tab. 5), zeigt, daß mit der Ausnahme von Norfloxacin keine Tendenz des gehäuften Auftretens großer/sehr großer Abweichungen bei bestimmten Bakterienarten oder Antibiotika feststellbar ist. Darüber hinaus ist zu bemerken, daß die Anzahl sehr großer Abweichungen im Gesamtergebnis 7,3\% (Tab. 3) beträgt. Bei einzelnen Keim-Antibiotikum-Kombinationen (ohne Berücksichtigung von Norfloxacin) ist diese Abweichung bis zu 50\% (Ceftriaxon/Proteus vulgaris).
Tab. 4: Unterschiede der Empfindlichkeitsbestimmung verschiedener Bakterien mit unterschiedlichen Antibiotika mit Hilfe des COBAS-Bact im Vergleich zur Mikrodilutionsmethode (DIN 58940). ( $n=$ Gesamtzahl der untersuchten Bakterienstämme)

\begin{tabular}{lrrrr} 
Antibiotikum & $\mathbf{n}$ & \multicolumn{3}{c}{ Abweichungen in \% } \\
\cline { 3 - 5 } & & kleine & große & sehr große \\
\hline Ampicillin & 341 & 12,6 & 0,3 & 13,8 \\
Piperacillin & 363 & 32,0 & 0,6 & 9,9 \\
Ceftazidim & 283 & 9,5 & 12,4 & 1,1 \\
Ceftriaxon & 282 & 14,2 & 0,7 & 7,4 \\
Gentamicin & 367 & 38,4 & 0,8 & 3,5 \\
Cotrimoxazol & 338 & 4,4 & 2,1 & 4,7 \\
Amoxy/Clav. & 320 & 16,3 & 3,8 & 8,4 \\
Cefuroxim & 344 & 38,1 & 1,7 & 3,5 \\
Latamoxef & 274 & 20,8 & 0,0 & 0,0 \\
Cefazolin & 339 & 12,1 & 0,9 & 2,4 \\
Norfloxacin & 368 & 1,6 & 0,5 & 25,8 \\
Cefoperazon. & 278 & 27,7 & 5,0 & 10,1 \\
Cefsulodin & 26 & 80,8 & 0,0 & 0,0 \\
Penicillin & 49 & 18,4 & 0,0 & 2,0 \\
Oxacillin & 69 & 2,9 & 1,4 & 5,8 \\
& & & &
\end{tabular}

Die Untersuchungen zur Präzision der Empfindlichkeitsbestimmung mit dem COBAS-Bact zeigten, daß bei der Untersuchung von zwei Stämmen von E. coli, zwei Stämmen von C. freundii und einem Stamm von Klebsiella spec. in jeweils sechs parallelen Ansätzen nur bei der Kombination C. freundii/Ampicillin größere Abweichungen zu beobachten waren, dergestalt daß einmal empfindlich und fünfmal resistent, bzw. viermal empfindlich und zweimal resistent als Beurteilung ausgegeben wurde. Bei der Empfindlichkeitsbestimmung von 12 verschiedenen Bakterienstämmen gegenüber sieben Antibiotika an fünf aufeinanderfolgenden Tagen konnte allerdings kein so einheitliches Bild gefunden werden (Tab.6). Es zeigte sich, daß bei allen untersuchten Keimen zumindest in einer Kombination Abweichungen um zwei Beurteilungsstufen auftreten.

\section{Diskussion}

Das COBAS-Bact-System stellt ein automatisiertes System zur Identifikation von Enterobacteriaceae und zur Empfindlichkeitsprüfung von schnellwachsenden $\mathrm{Mi}$ kroorganismen dar $(2,8,12)$. Untersuchungen verschiedener Autoren $(4,6,9,10)$ zur Antibiotika-Empfindlichkeitsbestimmung zeigten eine wesentliche Übereinstimmung (volle Übereinstimmung und kleinere Abweichungen) von mehr als $90 \%$. $\mathrm{Da}$ sich die bekannten Untersuchungen nur auf Vergleiche mit Agardiffusionsund Agardilutionsmethoden beschränkten, sollten im Rahmen dieser Studie Vergleiche mit der MHK-Bestimmung nach der Bouillon-Dilutionsmethode (3) gezogen werden. Weiterhin wurde das Untersuchungsgut so ausgewählt, daß die MHK viẹler Stämme möglichst nahe an den „breakpoints” lag.

Die Ergebnisse zeigen, daß die hier erhobenen Befunde mit einer totalen Übereinstimmung von $71,1 \%$ und einer wesentlichen Übereinstimmung (totale Übereinstimmüng plus kleinere Abweichungen) von $90,7 \%$ doch deutlich geringer sind als die bisher veröffentlichten $\mathrm{Er}$ gebnisse von Dupuis (4) mit $90,0 \%$ und $95,5 \%$. Schaper und Woratz (9) mit $89 \%$ und $99 \%$ und Schoerner et al (10) mit $90 \%$ und $97 \%$. Diese Unterschiede sind wahrscheinlich auf Unterschiede in der Antibiotika-Empfind- 
lichkeitsverteilung der untersuchten Bakterienpopulationen zurückzuführen. So waren in der Untersuchung von Schaper und Woratz (9) 95\% der untersuchten Bakterienstämme (bezogen auf die Referenzmethode) gegenüber Gentamicin empfindlich, während in der hier vorliegenden Untersuchung nur 46\% (Tab. 2) der Bakterienstämme gegenüber Gentamicin empfindlich waren. Daten über die Antibiotika-Empfindlichkeit der untersuchten Bakterien in Hinblick auf die Referenzmethode liegen aus den anderen Untersuchungen leider nicht vor. Ein weiterer direkter Vergleich der Ergebnisse ist aufgrund der unterschiedlichen Zusammensetzung der untersuchten Bakterienpopulationen sowie der unterschiedlichen untersuchten Antibiotika nicht möglich.

Es ist allerdings zu bemerken, daß bei einigen Keimspezies oder Antibiotika sehr große Abweichungen von über $10 \%$ beobachtet wurden (Tab.3, 4). Ähnlich hohe Abweichungen wurden auch von Schaper und Wolratz (9) bei einigen Kombinationen gefunden. Diese hohen Abwei- chungen sind zu einem Teil auf das Auswerteprogramm des COBAS-Bact zurückzuführen. So sollen die schlechten Ergebnisse der Empfindlichkeitsprüfung mit Norfloxacin (Tab. 4, 5) durch eine Änderung der Software nicht mehr auftreten (Angaben des Herstellers). Ein größeres Problem ist allerdings in dem Auftreten sehr großer und großer Abweichungen bei einzelnen Keim/AntibiotikaKombinationen zu sehen (Tab. 5). Die Ursachen für diese einzelnen Abweichungen sind im Einzelfall nicht bekannt. Es ist allerdings in Betracht zu ziehen, daß, bedingt durch die kurze Meßzeit des Gerätes ( 5 Std.), im Gegensatz zur Inkubation für $18 \mathrm{Std}$. Vorgänge wie Induktion von $\beta$ Laktamase-Produktion, sowie Selektion von langsamer wachsenden resistenten Subpopulationen nur in einigen Fällen durch Analyse der Wachstumskurven erfaßt werden können, wodurch allerdings der zeitliche Aufwand erheblich vergrößert wird.

Gründe für die schlechte Tag-zu-Tag-Reproduzierbarkeit der Ergebnisse (Tab.6) können zur Zeit noch nicht ange-

Tab.5: Darstellung der großen und sehr großen Abweichungen der Empfindlichkeitsprüfung mit dem COBAS-Bact im Vergleich zur Mikrodilution bei einzelnen Keim-Antibiotikum-Kombinationen

\begin{tabular}{|c|c|c|c|c|c|c|c|c|c|c|}
\hline \multirow[t]{2}{*}{ Bakterien } & \multirow[t]{2}{*}{$\mathbf{n}$} & \multicolumn{9}{|c|}{ große/sehr große Abweichungen in \% } \\
\hline & & $\begin{array}{l}\text { Ampi- } \\
\text { cillin }\end{array}$ & $\begin{array}{l}\text { Piper- } \\
\text { acillin }\end{array}$ & $\begin{array}{l}\text { Cefta- } \\
\text { dizim }\end{array}$ & $\begin{array}{l}\text { Genta- } \\
\text { micin }\end{array}$ & $\begin{array}{l}\text { Amoxy/ } \\
\text { Clav. }\end{array}$ & $\begin{array}{l}\text { Cefur- } \\
\text { oxim }\end{array}$ & $\begin{array}{l}\text { Norflo- } \\
\text { xacin }\end{array}$ & $\begin{array}{l}\text { Cefo- } \\
\text { perazon }\end{array}$ & $\begin{array}{l}\text { Cef- } \\
\text { triaxon }\end{array}$ \\
\hline $\begin{array}{l}\text { E. coli } \\
\text { Klebsiella spec. } \\
\text { Serratia spec. } \\
\text { Morganella }\end{array}$ & $\begin{array}{l}48 \\
29 \\
29\end{array}$ & $\begin{array}{ll}0,0 / & 8,3 \\
0,0 / & 3 ; 4 \\
0,0 / & 0,0\end{array}$ & $\begin{array}{ll}4,3 / & 2,2 \\
0,0 / & 6,9 \\
0,0 / & 0,0\end{array}$ & $\begin{array}{r}29,2 / 0,0 \\
3,4 / 0,0 \\
34,5 / 0,0\end{array}$ & $\begin{array}{ll}0,0 / 2,1 \\
0,0 / 6,9 \\
0,0 / 17,2\end{array}$ & $\begin{array}{rr}14,6 / 6,3 \\
6,9 / 0,0 \\
0,0 / 0,0\end{array}$ & $\begin{array}{ll}4,2 / & 0,0 \\
0,0 / & 0,0 \\
0,0 / & 0,0\end{array}$ & $\begin{array}{ll}0,0 / & 0,0 \\
6,9 / & 0,0 \\
0,0 / 17,2\end{array}$ & $\begin{array}{r}21,3 / 0,0 \\
0,0 / 31,0 \\
6,9 / 17,2\end{array}$ & $\begin{array}{ll}0,0 / & 8,3 \\
0,0 / & 3,4 \\
0,0 / & 6,9\end{array}$ \\
\hline $\begin{array}{l}\text { morganii } \\
\text { Enterobacter }\end{array}$ & 27 & $0,0 / 25,9$ & $0,0 / 7,7$ & $15,4 / 0,0$ & $0,0 / 0,0$ & $0,0 / 18,5$ & $0,0 / 7,4$ & $0,0 / 0,0$ & $0,0 / 3,8$ & $0,0 / 0,0$ \\
\hline $\begin{array}{l}\text { cloacae } \\
\text { Citrobacter }\end{array}$ & 30 & $0,0 / 20,0$ & $0,0 / 3,3$ & $0,0 / 0,0$ & $0,0 / 0,0$ & $0,0 / 20,0$ & $0,0 / 0,0$ & $0,0 / 6,7$ & $0,0 / 13,3$ & $0,0 / 0,0$ \\
\hline $\begin{array}{l}\text { freundii } \\
\text { Proteus }\end{array}$ & 30 & $0,0 / 6,7$ & $0,0 / 10,0$ & $3,3 / 3,3$ & $6,7 / 6,7$ & - $3,3 / 13,3$ & $0,0 / 0.0$ & $0,0 / 3,3$ & $0,0 / 23,3$ & $0,0 / 3,3$ \\
\hline $\begin{array}{l}\text { mirabilis } \\
\text { Proteus }\end{array}$ & 17 & $0,0 / 6,3$ & $0,0 / 35,3$ & $0,0 / 0,0$ & $0,0 / 0.0$ & $0,0 / 5,9$ & $0.0 / 0.0$ & $0,0 / 0,0$ & $0,0 / 5,9$ & $0,0 / 5,9$ \\
\hline $\begin{array}{l}\text { vulgaris } \\
\text { Pseudomonas }\end{array}$ & 30 & $0,0 / 6,7$ & $0,0 / 23,3$ & $0,0 / 0,0$ & $0,0 / 0,0$ & $0,0 / 10,0$ & $0,0 / 20,0$ & $0,0 / 0,0$ & $0,0 / 0,0$ & $0,0 / 50,0$ \\
\hline $\begin{array}{l}\text { aeruginosa } \\
\text { Staph. aureus } \\
\text { Strep. faecalis } \\
\text { Strep. agalactiae }\end{array}$ & $\begin{array}{l}26 \\
34 \\
36 \\
15\end{array}$ & $\begin{array}{ll}0,0 / 0,0 \\
0,0 / . .0,0\end{array}$ & $\begin{array}{l}0,0 / \quad 8,3 \\
0,0 / 29,4 \\
0,0 / 0,0 \\
0,0 / 0,0\end{array}$ & $0,0 / 7.7$ & $\begin{array}{ll}3,8 / & 0,0 \\
0,0 / & 0,0 \\
0,0 / & 2,9 \\
0,0 / & 0,0\end{array}$ & $\begin{array}{l}0,0 / 11,8 \\
2,9 / 0,0 \\
6,7 / 0,0\end{array}$ & $\begin{array}{l}0,0 / 42,3 \\
0,0 / 5,9 \\
0,0 / 2,9 \\
0,0 / 0,0\end{array}$ & $\begin{array}{l}0,0 / 73,5 \\
0,0 / 97,2 \\
0,0 / 93,3\end{array}$ & $0,0 / 4,2$ & $0,0 / 4,0$ \\
\hline
\end{tabular}

Tab.6: Ergebnisse der Empfindlichkeitsprüfung verschiedener Bakterienstämme an fünf aufeinanderfolgenden Tagen mit dem COBASBact

\begin{tabular}{|c|c|c|c|c|c|c|c|c|c|c|c|c|c|c|c|c|c|c|c|c|c|}
\hline \multirow[t]{2}{*}{ Stamm } & \multicolumn{3}{|c|}{ Ampi. } & \multicolumn{3}{|c|}{ Pipera. } & \multicolumn{3}{|c|}{ Coftazid. } & \multicolumn{3}{|c|}{ Ceftriax. } & \multicolumn{3}{|c|}{$\begin{array}{l}\text { Amoxy/ } \\
\text { Clav. }\end{array}$} & \multicolumn{3}{|c|}{ Cefurox. } & \multicolumn{3}{|c|}{ Cefopera } \\
\hline & $\mathbf{E}$ & (E) & $\mathbf{R}$ & $\mathbf{E}$ & (E) & $\mathbf{R}$ & $\mathbf{E}$ & (E) & $\mathbf{R}$ & $\mathbf{E}$ & (E) & $\mathbf{R}$ & $\mathbf{E}$ & (E) & $\mathbf{R}$ & $\mathbf{E}$ & (E) & $\mathbf{R}$ & $E$ & (E) & $R$ \\
\hline $\begin{array}{l}\text { E. coli U4032 } \\
\text { E. coli U2012 } \\
\text { C. freundii } 210 \\
\text { C. freundii } 342 b \\
\text { P. mirabilis U2191 } \\
\text { P. mirabilis U4752 } \\
\text { P. vulgaris } 44 \\
\text { P. vulgaris U4917 } \\
\text { Klebsiella } 2682 \\
\text { Kiebsiella U3972 } \\
\text { Serratia U1581 } \\
\text { Serratia } 1009 \text { a }\end{array}$ & $\begin{array}{l}5 \\
3 \\
2\end{array}$ & 更 & $\begin{array}{l}5 \\
2 \\
2 \\
5 \\
4 \\
5 \\
4 \\
5 \\
5 \\
5 \\
5\end{array}$ & $\begin{array}{l}5 \\
5 \\
5 \\
2 \\
4 \\
4 \\
5 \\
2 \\
2\end{array}$ & $\begin{array}{l}1 \\
1 \\
2\end{array}$ & $\begin{array}{l}5 \\
2 \\
1\end{array}$ & $\begin{array}{l}5 \\
5 \\
5 \\
4 \\
4 \\
2 \\
4 \\
5 \\
5 \\
5 \\
3 \\
3\end{array}$ & . & $\begin{array}{l}1 \\
1 \\
2 \\
1\end{array}$ & $\begin{array}{l}5 \\
5 \\
5 \\
5 \\
5 \\
2 \\
4 \\
5 \\
5 \\
5 \\
5 \\
3\end{array}$ & $\begin{array}{l}1 \\
1\end{array}$ & 1 & $\begin{array}{l}3 \\
5 \\
5 \\
1 \\
\\
4 \\
5 \\
4 \\
2 .\end{array}$ & $\begin{array}{l}4 \\
4 \\
1\end{array}$ & $\begin{array}{l}1 \\
2 \\
5 \\
5\end{array}$ & $\begin{array}{l}1 \\
5 \\
4 \\
2 \\
4\end{array}$ & $\begin{array}{l}4 \\
1 \\
2\end{array}$ & $\begin{array}{l}1 \\
1 \\
4 \\
5 \\
1 \\
\\
5 \\
5\end{array}$ & $\begin{array}{l}5 \\
5 \\
5 \\
5 \\
3 \\
3 \\
4 \\
5 \\
5 \\
4\end{array}$ & $\begin{array}{l}1 \\
1 \\
1\end{array}$ & $\begin{array}{l}1 \\
3 \\
1\end{array}$ \\
\hline
\end{tabular}


geben werden. Es besteht die Möglichkeit, daß hier Fehlbeschickungen der Testblättchen oder Unterschiede in der Dichte der Keimsuspension Auswirkungen zeigen. Für eine kritische Betrachtung weiterer Vor- und Nachteile des COBAS-Bact-Systems sei auf die Veröffentlichung von Schoerner et al. (10) verwiesen.

\section{Danksagung}

Wir danken der Firma Hoffmann La Roche (Grenzach) für die freundliche Überlassung eines COBAS-Bact-Gerätes zur Durchführung dieser Studie.

Anschrift des Verfassers:

Dr. Karl-Heinz Büscher

Medizinische Mikrobiologie und Immunologie

Ruhr-Universität Bochum, MA 01

Postfach 102148

4630 Bochum
Schriftum:

1. ARNI P DOLLENMEIER, P. MOLLER: D.: Automated modification of the Ames test with COBAS Bact. Mutation Res. 144, 137- 140 (1985).

2. BALOWS, A.: COBAS Bact: The centrifugal approach to clinical bacteriology. In: Rapid methods and automation in microbiology and immunology. K.-O. Habermeh (editof). Springer Verlag. Berlin, Heidelberg. pp. 297-304 (1985).

3. DIN-Norm Nr.58940, 1 -6 Methoden zur Empfindlichkeitsprûfung von bakteriellen Krankheitserregern (außer Mycobakterien) gegen Chemotherapeutika. Beuth Verlag Berlin.

4. DUPUIS, G.: Evaluation of the COBAS Bact antimicrobial susceptibility testing system. Eur. J. Clin. Microbiol. 4, 119-122 (1985).

5. HABERMEHL, K.-O. (editor): Rapid methods and automation in microbiology and immunology. Springer Verlag, Berlin, Heidelberg (1985).

6. HEIZMANN, W., WERNER, H., HEILMANN, F.: Evaluation of the COBAS Bact automated system for susceptibility testing of enterobacteriaceae. Pseudomonas aeru ginosa, and Enterococcus faecalis to azlocillin, mezlocillin, and ciprofloxacin compared to NCCLS and DIN standards. Infection 16, 63/71-76-68 (1988).

7. LENNETTE, E. H., BALOWS, A., HAUSLER, W. J., SHADOMY, H. J. (editors) Manual of clinical microbiology. American Society for microbiology. Washington D.C. (1985).

8. MARTIN, R.: Blutkulturtechnik und bakteriologische Schnelldiagnostik. Swiss Med. 6. $51-58$ (1984)

9. SCHAPER, B., WORATZ, H.: Vergleich eines hochmechanisierten Systems zu Empfindlichkeitsprüfung von Bakterien mit dem Agardiffusionstest (DIN 58940) Lab.med. 8, $391-395$ (1984).

10. SCHOERNER, C., BOLTZE, H.-J., ROLLINGHOFF, M.: Antibiotic susceptibility testing with the COBAS Bact-System. Äratl. Lab., in press.

11. TILTON, R. C. (editor): Rapid methods and automation in microbiology. American Society for Microbiology. Washington D.C. (1982).

12. WENGER, A., TISSIERES L. J., PRAPLAN, F. R., KAMM, W. R., BILLE, J. COBAS-Bact system for identification of members of the family enterobacteriaceae in 4 h $20 \mathrm{~min}$. J. Clin. Microbiol. 25, 61-66 (1987). 


\section{Die höchst zuv eillässige Fammilie vom Ellektrolyt-A malysatorem.}

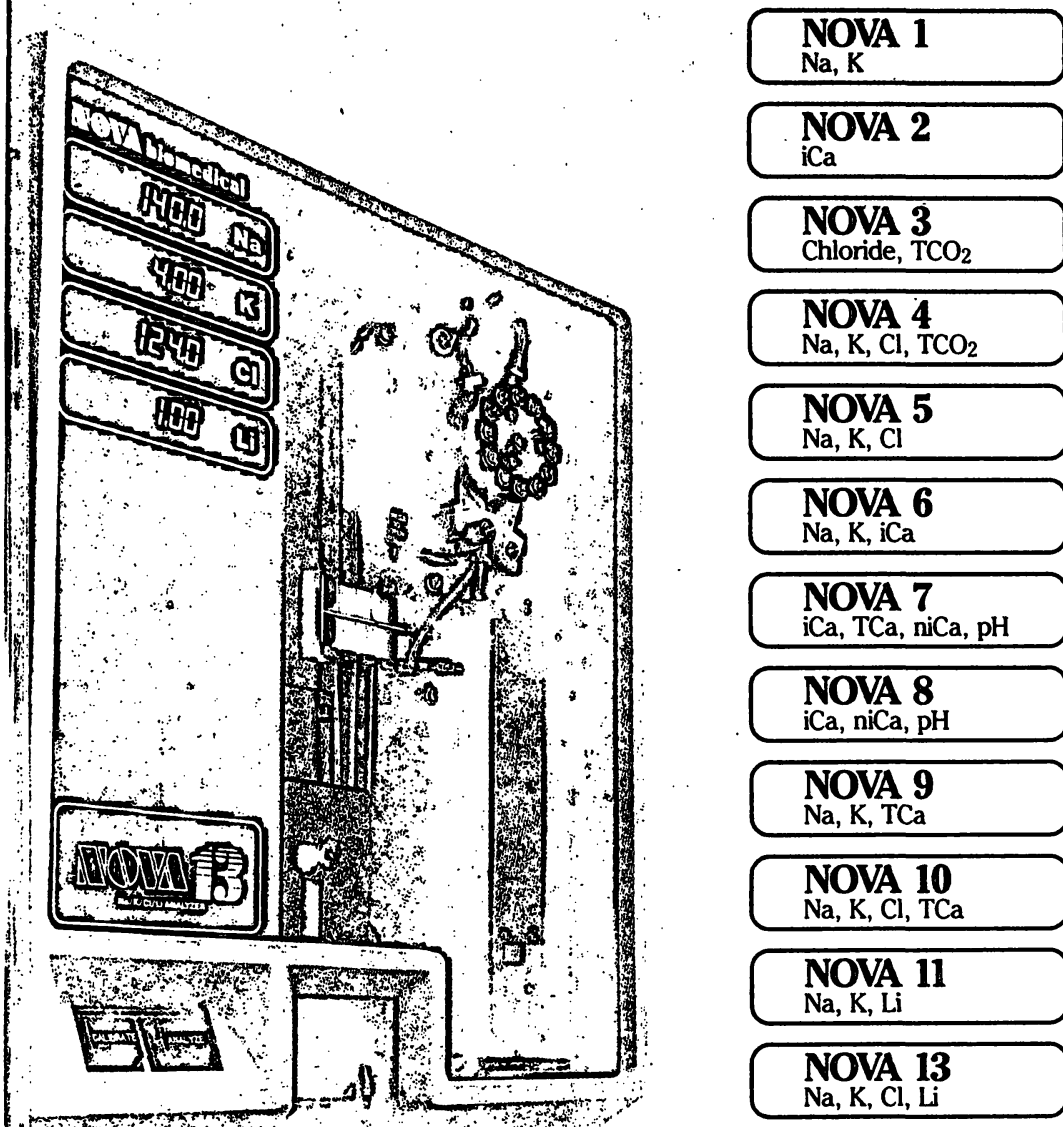

NOVA hat mit über 7000

Elektrolytanalysatoren mehr

Geräte in mehr Laboratorien

plaziert als irgendjemand .

anderes.

\section{Übereinstimmend nennen} unsere Kunden als wichtigsten Grund ihrer Kaufentscheidung die Zuverlässigkeit der

NOVA-Analysatoren.

NOVA-Analysatoren sind zuverlässig. Kein anderes Gerät hat sich aufgrund seiner Konzeption in über zehn Jahren ähnlich bewährt. Über 7000 Benutzer bestätigen dies im täglichen Einsatz ihres Gerätes. Wir sind stolz auf den Vertrauensbeweis unserer Kunden. Kontinuierliche Weiterentwicklungen, größte Produktpalette und fortschrittlichste ISE-Technologie zcigen die Ausnahmestellung von NOVA in diesem Marktsegment.

Zögern Sie nicht, für Ihre nächste Entscheidung die Leistung und Zuverlässigkeit von NOVA-Geräten zu testen.

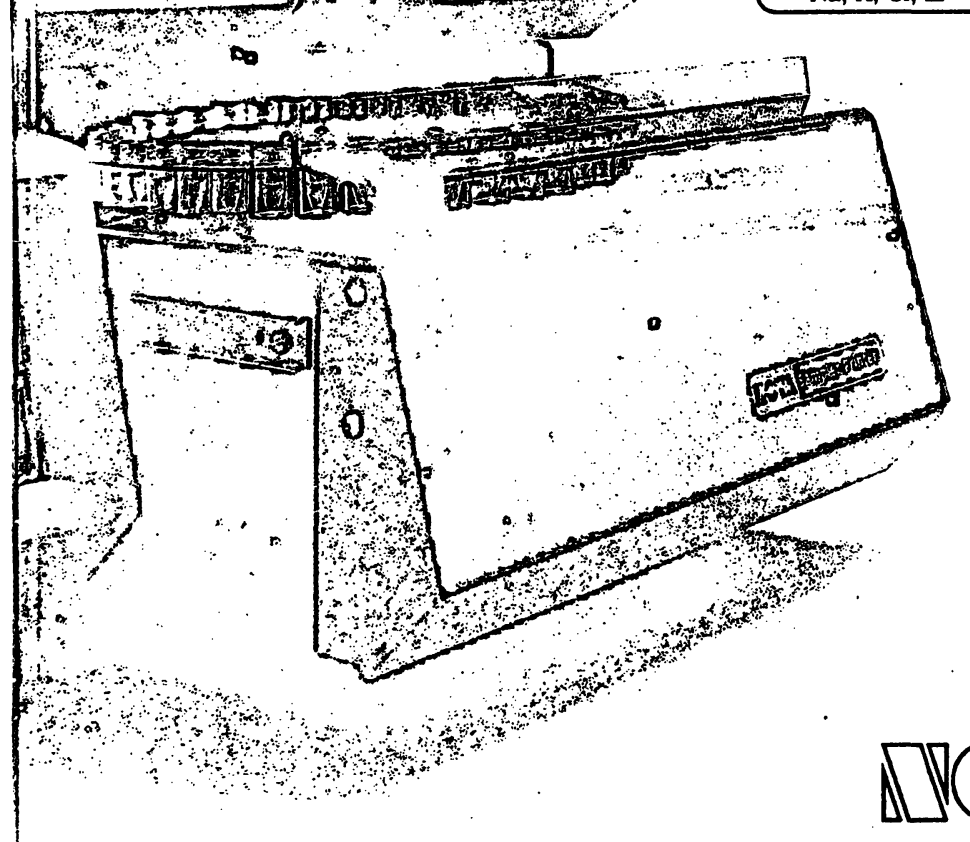




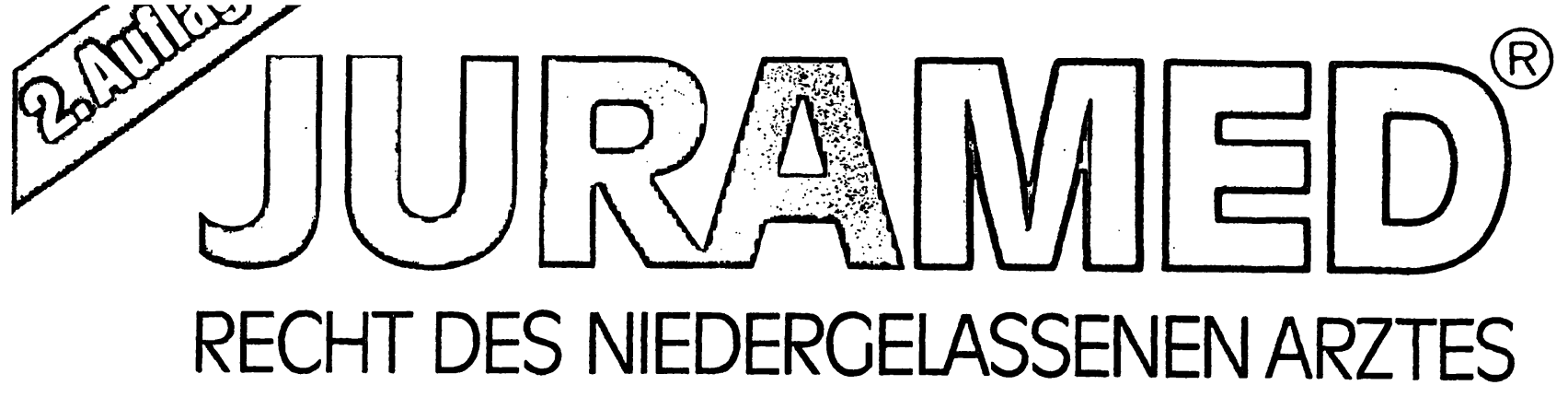

\section{Was ist JURARAED?}

JURAMED ist ein praxisnahes Werk über das Recht des niedergelassenen Arztes, zusammengestellt von anerkannten Fachleuten, die wissen, wo den niedergelassenen Arzt (juristisch) der Schuh drückt.

Die übersichtliche Gestaltung des Werkes und ein hervorragend gegliedertes Register ermöglichen dem Ratsuchenden, in kürzester Zeit fündig zu werden. Die hier jeweils angebotene prägnante Problemlösung, auch auf arbeitsrechtlichem Gebiet, erlaubt es dem Benutzer, zu agieren und nicht zu reagieren.

Nentwig, W. M.: JURAMED Recht des niedergelassenen Arztes. Loseblattwerk im Sammelordner, 2. Auflage, 98,- DM, einmal jährlich erscheinende Nachtragslieferung (Seitenpreis 0,29 DM) ISBN 3-87409-003-5.

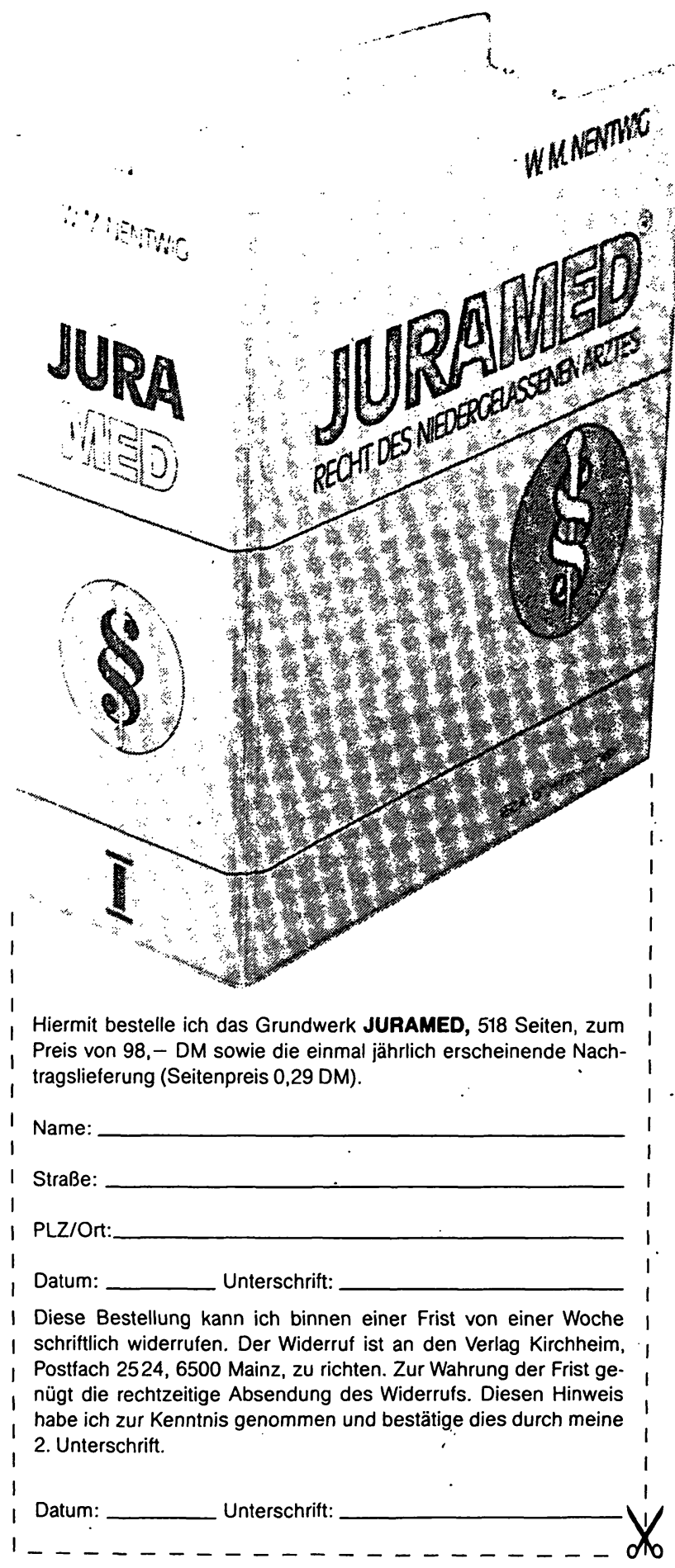

Warum ist JURARED immer aktuell?

Da es sich um eine Loseblattsammlung handelt, sind die häufig auftretenden Änderungen in Gesetz und Rechtsprechung in kürzester Zeit Bestandteile des Werkes und stehen aktuell zur Verfügung.

Die Beschränkung auf die wesentlichen Kernprobleme der jeweiligen Fallgestaltung und deren griffige Darstellung erlaubt es auch dem unter chronischer Zeitnot leidenden Leser, schnell umfassend informiert zu sein.

In einer Zeit, in der sich der niedergelassene Arzt mehr und mehr mit rechtlichen Problemen konfrontiert sieht, ist JURAMED ein unentbehrlicher Begleiter.

\section{KETRCC HHEIM}

Postfach 2524, 6500 Mainz 


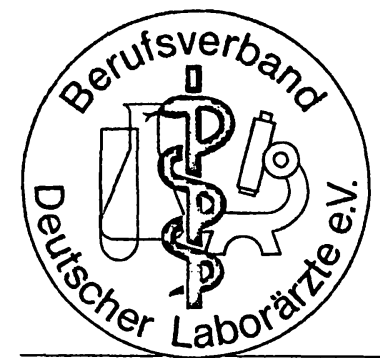

Mitteilungen des

BERUFSVERBAND

DEUTSCHER LABORÄRZTE e.V.

\section{Bericht von der 13. Tagung der Deutschen Gesellschaft für Parasitologie}

Vom 22. bis 25. März 1988 tagte die DGP in Neuchâtel/ Schweiz. Über einige für die Humanmedizin relevante Kurzvorträge soll hier berichtet werden.

Die Diagnose der zerebralen Toxoplasmose ist klinisch mittels Computertomogramm, Kernspintomogramm und Gehirnbiopsie möglich. Der Antikörpernachweis wird jedoch weitgehend als unzuverlässig angesehen, da in der üblichen Routineserologie die Antikörpertiter im niedrigen Bereich verbleiben. Durch Untersuchüngen entsprechender Seren aus dem Münchener Gebiet konnte jedoch gezeigt werden, daß bei gleichzeitiger Anwendung mehrerer Verfahren eine Serodiagnose in vielen Fällen möglich ist. Der Sabin-Feldman-Test (bzw. IFT) und die Direkte Agglutination eignen sich als gute Screening-Verfahren, die KBR und die indirekte Hämagglutination bieten zusätzliche Informationen bei schwach positiven Titern. In einzelnen Fällen gelang auch der Nachweis von IgMAntikörpern mittels Enzymimmunoassay (EIA). Diese Methode kommt auch generell bei der Serodiagnose der Toxoplasmose zum Einsatz. Die Sensitivität und Spezifität beim EIA-IgG beträgt ca. 97\%. Der indirekte Immunfluoreszenztest auf spezifisches $\operatorname{lgM}$ ist wenig empfindlich und spezifisch und sollte daher durch den EIA-IgM ersetzt werden. Schwierig ist bisher auch der Nachweis der Augentoxoplasmose. Am Kaninchen ist nun ein neues Verfahren erfolgreich erprobt worden. Fluorescein-markiertes Anti-Toxoplasma-lgG wurde in die Ohrvene injiziert. Toxoplasma-bedingte Läsionen am Augenfundus konnten dann mittels Fluoreszenz-Angiographie und kontrastverstärkenden Bildbearbeitungstechniken dargestellt werden.

Kryptosporidien sind vor allem als Durchfallserreger bei immundefizienten Patienten bekannt. Diese Parasiten sind wenig wirtsspezifisch, und daher gelten Haustiere, vor allem Kälber, als Infektionsquellen. Es konnte nun nachgewiesen werden, daß auch Katzen (zu 4,25\%) und Hunde $(0,72 \%)$ Träger von Kryptosporidien sind. Da die Befallsintensität jedoch äußerst niedrig war, ist die epidemiologische Bedeutung dieser Tiere als relativ gering einzustufen.

Die diagnostische Zuverlässigkeit serologischer Teste wird wesentlich durch die verwendeten Antigene bestimmt. Mittels biochemischer Methoden gelang es jetzt, Proteine aus Schistosomen (Bilharzien) zu isolieren, die gattungsspezifisch sind. Mit fast $100 \%$ Sensitivität und
Spezifität können damit Schistosoma mansoni-, S. haematobium- und S. japonicum-Infektionen nachgewiesen werden.

Als hoch endemisches Gebiet für die alveoläre Echinokokkose (E. multilocularis) gilt die Schwäbische Alb. Dort erwiesen sich durchschnittlich 18,3\% der untersuchten Füchse als infiziert, wobei die Kreise Tuttlingen und Zollern-Alb mit bis zu 29,1\% an der Spitze lagen. Weitere Vorkommen des Parasiten befinden sich besonders im Schwarzwald, im Odenwald, bei Memmingen und am Starnberger See. Durch Verzehr von mit EchinokokkenEiern kontaminierten Waldfrüchten besteht eine Ansteckungsgefahr für den Menschen.

Überträger der Borrelia burgdorferi (Lyme-Krankheit) ist die Zecke Ixodes ricinus. Im Siebengebirge bei Bonn, einem Endemiegebiet, konnte nachgewiesen werden, daß die Zeckendichte im Zusammenhang mit bestimmten Pflanzengemeinschaften (z.B. Frauenfarn) steht. Mit steigender Dichte der Zecken nimmt die Zahl derer, die infiziert sind, überproportional zu. Die Durchseuchung der Anthropoden lag zwischen 0 und 30\%. Untersuchungen in der Schweiz lassen vermuten, daß die Übertragung der Borrelien nur selten von adulten Zeckenweibchen auf die Eier (transovariell) möglich ist, da Larven nur zu $3,06 \%$, an Nagetieren und Vögeln saugende Nymphen zu $11,62 \%$ und geschlechtsreife Zecken zu 14,54\% Träger des Erregers waren (transstadiale Übertragung).

Im Mittelpunkt der Tagung standen Berichte über die Anwendung gentechnologischer Verfahren in der parasitologischen Grundlagenforschung, die in naher Zukunft auch zu praxisrelevanten Ergebnissen führen werden.

K. Janitschke, Berlin

\section{Bitte vormerken:}

Herbsttagung des Berufsverbandes

Deutscher Laborärzte vom 28. bis 30. Oktober 1988 in Bad Nauheim. Thema: Immunopathien 


\section{Jahrestagung 1988 der Deutschen Gesellschaft für Infektiologie}

Wie in den vergangenen Jahren führte die Deutsche Gesellschaft für Infektiologie ihre 14. Jahrestagung vom 12. bis 14. Mai 1988 auf der Reisensburg bei Günzburg durch.

Der erste Halbtag befaßte sich mit chronisch-progressiven Infektionen des Zentralnervensystems. Zuerst wurde über wenig bekannte Virusinfektionen berichtet, so die Borna-Krankheit (A. Mayr, München), einer 1894 in Borna/Sachsen erstmals beschriebenen, als Pferdeseuche bekannten Virusinfektion, die auch beim Schaf und Kaninchen vorkommt, wobei noch vollkommen offen ist, ob sie auf den Menschen übergehen kann.

Bei Schaf und Ziege ist das zu den Lentivirinae gehörende Medi-Visna-Virus von Bedeutung (O. C. Straub, Tübingen), von dem es einen pneumotropen Typ (Medi) und einen neurotropen Typ (Visna) gibt.

In seinem Beitrag „Zielzellen für HIV im Zentralnervensystem" zeigte $V$. Erfle, München, zwei mögliche Wege auf; über die das HIV in das ZNS gelangen kann. Eine Möglichkeit ist, daß infizierte Makrophagen die Bluthirnschranke überwinden, der andere Weg wäre, daß Monozyten/Makrophagen Endothelzellen infizieren, die wiederum die Infektion an Astrozyten weitergeben. In vivo konnten im Zentralnervensystem HIV-infizierte Makrophagen (mehrkernige Riesenzellen) und vereinzelt Astrozyten, Oligodendroglia, Neurozyten und Endothelzellen gefunden werden. Bemerkenswert ist, daß sich virusexprimierende Zellen nicht teilen.

Eine sehr selten auftretende Erkrankung ( 1 bis 2 Neuerkrankungen $/ 1$ Million Einwohner) ist die Creutzfeld-Jakobsche Krankheit, über deren klinisches Bild $F$. Krull, Köln, sprach. In einer Video-Aufzeichnung konnte er eindrucksvoll die generalisierten Myoklonien demonstrieren. Ein Erreger konnte bisher elektronenmikroskopisch nicht dargestellt werden. Bekannt ist eine iatrogene Übertragung von Mensch zu Mensch, z. B. bei Operationen.

Bei der tertiären Neuroborreliose stellte $R$. Ackermann, Köln, die Parallele zur tertiären Lues heraus. Die mit Erythem, Schmerzen und neurologischen Ausfällen im Sinne einer asymmetrischen Meningopolyneuritis einhergehende Primärerkrankung klingt in 4 bis 5 Monaten auch ohne Therapie ab. Nach einem Latenzstadium, das bei 44 . Fällen mit einem Durchschnittsalter von 45 Jahren ( 7 bis 79 Jahre) durchschnittlich 2,8 Jahre (7 Monate bis 12 Jahre) dauerte, kam es zu einer progressiven Enzephalomyelitis, die als spinaler Typ (MS-like) oder als cerebraler Typ (multifokale Enzephalitis) auftrat. Während Eiweiß und Zellzahl im Liquor normal sein können, ist der Antikörpertiter gegen Borrelien pro Mikrogramm $\operatorname{lgG}$ im Liquor immer um 2 bis 5 Stufen höher als im Serum. Dies trifft auch zu für eine tertiäre Polyneuritis oder eine latente Neuroborreliose.

Über den Fall einer Manifestation des Morbus Whipple am Zentralnervensystem berichtete $S$. Bamborscheke, Köln. 4 Jahre nach Bestehen rezidivierender gastrointestinaler Symptome kam es erstmals zu neurologischen Erscheinungen mit Verwirrtheit und einem Hydrozephalus internus. Die Diagnose wurde gestellt durch eine Dünn- darmbiopsie, bei der sich morphologisch Makrophagen mit Speicherung nachweisen ließen. Eine bakterielle Genese wird diskutiert; dafür spricht auch das Ansprechen auf die Therapie mit Chloramphenicol oder Minocyclin und eine länger dauernde Nachbehandlung mit Cotrimoxazol. Isoliert wurden jedoch die verschiedensten Erreger wie Corynebakterien, Hämophilus, alphahämolytische Streptokokken, Lactobacillen, Aktinomyces. In der Diskussion wurde deshalb noch einmal herausgestellt, daß die Erregerzüchtung irrelevant sei. Da häufig auch Arthralgien beim Morbus Whipple auftreten, sah Eichenlaub, München, wenn deren Ursache ungeklärt sei, auch darin eine Indikation zur Dünndarmbiopsie.

Die Listerien-Encephalitis (E. Dahne, München), die sich bei Mensch und Wiederkäuern findet, ist streng auf den Hirnstamm lokalisiert. Eine hämatogene Meningitis oder Meningo-Encephalitis kommt bei immunsupprimierten Patienten vor, bei Neonaten kommt es zu einer Septikämie. Die Infektionen erfolgen aus der Umwelt, nicht von Individium zu Individium. Im allgemeinen sind die Listerien reine Saprophyten. Als pathogen haben sich Listeria monocytogenes und weniger Listeria ivanovii gezeigt. Die Diagnose kann immunhistologisch am Paraffinschnitt gestellt werden. In der Diskussion wurde bemerkt, daß in der Vaginalschleimhaut der Erreger bis zu 2 Jahren persitieren und der Mensch gelegentlich Keimträger sein kann. Ein Pathogenitätsnachweis kann durch Genom- und Plasmidanalyse erfolgen.

Der Nachmittag befaßte sich unter dem Titel „,Onkogene Viren", vor allem 'mit molekular-biologischen Fragen. Während bei der viralen Infektionskrankheit eine Fortpflanzung der Viren erforderlich ist, ist dies bei der malignen Erkrankung nicht der Fall, da das Virus ja sonst die Tumorzelle zerstören würde.

G. Gross, Hamburg, sprach über die Klinik und Therapie humaner Papillomatosen und stellte heraus, daß es unter den mehr als 50 verschiedenen Papillomvirustypen bestimmte Typen gibt, die ein höheres Risiko der malignen Entartung bieten. So gehen Plantarwarzen, die ausschließlich vom Typ 1 hervorgerufen werden, nie in einen malignen Zustand über. Ein hohes Risiko bieten die HPVTypen 16 und 18, die beim Zervix-Karzinom gefunden werden. Die Übertragung erfolgt am häufigsten sexuell, was sich in einem Gipfel zwischen dem 20. und 30. Lebensjahr zeigt. Die Diagnose erfolgt durch Inspektion und Essigsäuretouschierung, sowie durch die HPVDNA-Typisierung mit Southernblot (32 p-HPV-DNA). Eine Behandlung mit Alpha- und Gamma-Interferon, die mit niedriger Dosierung über längere Zeit in 3 Zyklen erfolgen sollte, führt zu einer narbenlosen Rückbildung der Papillomatosen. Rezidive treten danach nicht auf. Die Behandlung führt zu einer Temperaturerhöhung etwa 4 Std. nach der Interferongabe in den ersten Tagen und zu Gelenkbeschwerden. Kein Erfolg tritt bei Patienten mit Immundefekten und Morbus Hodgkin ein.

„HPV Genexpression und zelluläre Kontrollmechanismen beim Zervixkarzinom" war das Thema des Vortrages von E. Schwarz, Heidelberg. Er führte aus, daß der Weg von der normalen Zelle über eine Infektion mit HPV 16/18 mit Viruspersistenz über eine Integration der HPV-DNA und 
eine Inaktivierung der zellulären Suppressororgane zur malignen Zelle führt. Nicht die Virusinfektion allein führt zum Tumor, den nur ein geringer Anteil der HPV 16-/ HPV 18-Infizierten entwickeln. Es besteht eine lange Latenzzeit von etwa 5 bis 25 Jahren.

"Leberzell-Karzinom und Hepatitis B-Virus" war das Thema von $H$. Will, Martinsried. Während $65 \%$ der Hepatitis-B-Infektionen subklinisch verlaufen und $100 \%$ ig ausheilen, tritt bei $25 \%$ eine akute Typ B-Hepatitis auf, bei den restlichen $10 \%$ entwickelt sich eine chronische Hepatitis, bei der virale DNS in das Chromosom integriert wird. Diese Träger einer chronischen Hepatitis haben ein 200 fach höheres Risiko an einem Leberkarzinom zu erkranken, als nicht an Hepatitis B Erkrankte. Für einen Zusammenhang spricht $u$.a., daß die Hepatitis B immer zuerst auftritt. Daß nur wenige chronisch Hepatitis B-VirusErkrankte ein Leberzell-Karzinom bekommen, spricht dafür, daß das Virus selbst nicht onkogen ist. Die überwältigende Mehrzahl der Leberkarzinome (über 90\%) enthält integrierte Virus DNS. Über die Rolle weiterer Faktoren, die für die Pathogenese des Karzinoms eine Rolle spielen, gibt es bisher nur Hypothesen. Hier wird der Alkohol angeführt; ob Aflatoxin eine Rolle spielt, ist noch nicht nachgeprüft.

EBV-korrelierte Tumorerkrankungen des Menschen ( $H$. Wolf, München) sind das Nasopharynx-Karzinom und das Burkitt-Lymphom, die in jeder Zelle EBV enthalten. Als zusätzlicher auslösender Faktor des z. B in Südchina häufig vorkommenden Nasopharynx-Karzinoms werden Nitrosamine und Bestandteile von Heilpflanzen angesehen. Hier bestehen auch Anstrengungen, einen Impfstoff, der neben dem HBS-Antigen auch EBV-Antigen enthält, herzustellen.

Großes Interesse fanden die Vorträge, die der aktuellen Infektiologie gewidmet waren. $M$. Just, Basel, beobachtete in 10 Jahren 144 invasive Hämophilus-Erkrankungen, davon 61 mit Meningitis (fast $70 \%$ Kinder unter 2 Jahren) und 44 Fälle von Epiglottitis (ca. 70\% der Kinder über 2 Jahre). Er berichtete über die Anti-Hämophilus-Impfung als Meningitis-Prophylaxe. Der Impfstoff wirkt nur protektiv bei Kindern über 2 Jahren. Ein neuer Impfstoff, der an Diphtherie-Toxoid gekoppelt ist und bei dem Impfungen mit 3, 4, 6 und 14 Monaten erfolgen, bietet in $84 \%$ Schutz gegen Hämophilus-Infektionen. $\mathrm{Er}$ selbst impfte 150 Kleinkinder mit 3, 4 und 5 Monaten und sah keine Nebenwirkungen. Nach der ersten Impfung gab es $63 \%$ und nach der dritten Impfung $89 \%$ Konverter mit einem schützenden Wert. Nach 15 bis 18 Monaten sollte eine Boosterung erfolgen.

H. Ch. Huber, München, der die Besiedelung der Gaumenmandeln mit fakultativ pathogenen Erregern bei 10jährigen in den Jahren 1984-1986 untersuchte und diese Untersuchung auf 5 Indikatorkeime ausdehnte (beta-hämolysierende Streptokokken, Enterobacteriaceae, Candida, Schimmelpilze, Pseudomonaden), unterteilte in lufthygienisch belastete Gebiete (Frankfurt/Main, Wiesbaden) und lufthygienisch nicht belastete Gebiete (Freiburg. Starnberg) und fand dabei in den nicht belasteten Gebieten deutlich weniger Indikatorkeime und nur die Hälfte deutlich vergrößerter Halslymphknoten; eine Einteilung und Klassifizierung, die in der Diskussion auf Widerspruch stieß.

S. Enenkel, Frankfurt, berichtete über Septikämien im Kindesalter und stellte dabei die Jahre 1974-1977 den Jahren 1984-1987 gegenüber. Während sich die Zahl der abgenommenen Blutkulturen mehr als verzehnfacht hatte, zeigte die Inzidenz der Septikämien mit 8,24 und
8,95 pro 1000 Patienten keine wesentliche Änderung. Die Häufigkeit von Staphylococcus aureus hatte abgenommen $(23 / 12 \%)$, während die von coagulasenegativen Staphylokokken von 8 auf $15 \%$ und die von Hämophilus von 4 auf $16 \%$ zugenommen hatte. Die Letalität, die $12,7 \%$ betrug, war am höchsten bei E. coli $(26 \%)$ und bei B-Streptokokken (33\%), wobei Begleiterkrankungen eine Rolle spielten.

Den von $R$. Schmidt, Köln, angekündigten Beitrag „Gefährdet eine Borrelien-Infektion des Blutspenders den Empfänger?" trug Ackermann vor. Unter 3157 Blutspendern fanden sich 86 (=3\%) Antikörper-positive. Von diesen konnten 47 untersucht werden. Bei 13 davon bestanden z.Zt. der Untersuchung oder in der Anamnese Krankheitszeichen, 15 konnten einen Zeckenstich angeben, und 33 waren einem Expositionsrisiko ausgesetzt. Die Isolierung von $B$. burgdorferi gelingt im Blut noch bis zu 5 Wochen, im Liquor bis zu 15 Wochen und in der Haut bis zu 10 Jahren nach der Infektion. Bei der Prüfung der Inaktivierung im Zitratblut zeigte sich, daß nach 96 Stunden kein Treponema pallidum mehr überlebte, aber nach 25 Tagen noch alle Proben mit Borrelia burgdorferi positiv waren, eine Probe noch nach 60 Tagen. Der Autor stellte die Frage, ob Personen, die in den letzten 3 Monaten einen Zeckenstich durchgemacht haben, von der Blutspende ausgeschlossen werden sollten.

B. Wilske, München, beschäftigte sich mit der immunochemischen Charakterisierung von Borrelia burgdorferi und konnte im Proteinprofil als konstante Proteine ein "Common antigen" mit $60 \mathrm{~K}$ und Flagellin mit $41 \mathrm{~K}$ als einheitliche Immunantwort feststellen. Daneben finden sich die variablen Proteine $\mathrm{OpB}$, OspA und $\mathrm{pC}$. Hierbei zeigen sich Unterschiede in Amerika und Europa. Während OspA in Amerika einheitlich vorkommt, ist es in Europa heterogen. Das $\mathrm{pC}$ ist in Amerika äußerst selten, dagegen in Europa in 50\% stark ausgeprägt. Das $60 \mathrm{~K}$ Common antigen zeigt eine starke Kreuzreaktivität mit Rü'ckfallfieber und Treponemen, während das Flagellin eine schwache Kreuzreaktivität mit Treponemen zeigt und daher als möglicher Kandidat für spätere serologische Teste in Frage kommt. Bisher konnten 7 Serotypen definiert werden.

In der kombinierten Anwendung von Interferon-beta und Interferon-gamma sieht J. Eisenburg, München, eine Möglichkeit der Behandlung der chronischen Hepatitis, die immer histologisch diagnostiziert werden sollte. Das $\mathrm{HBC}$ und $\mathrm{HBs}$ verschwinden immunhistochemisch nach der Behandlung, z. T. kommt es auch zu einer Serokonversion. Das neue Interferon ist weitgehend nebenwirkungsfrei. Anti-HIV sollte vor der Behandlung immer ausgeschlossen werden.

Cytomegalievirus ist der wichtigste und häufigste opportunistische Erreger im Gastrointestinaltrakt bei HIV-Infektionen ( $W$. Heise, Berlin). Der serologische Nachweis ist nicht aussagekräftig, es sollten immer Biopsien kulturell und immunhistochemisch untersucht werden. Bei 98 Patienten mit gastrointestinaler Symptomatik wurde in 28 Fällen bioptisch eine CMV-Infektion nachgewiesen. 18 dieser Patienten boten das Vollbild von AIDS, 4 einen AIDS-related Complex und 6 waren HIV-positiv. Von diesen Patienten konnte in 20 Fällen eine weitere Begleitinfektion, davon 10mal mit Mycobakterien, nachgewiesen werden. Klinisch fanden sich Ulcerationen im Ösophagus, Magen- und Darmtrakt.

Über. Nebenwirkungen durch Trimethoprim-Sulfamethoxazol, das als Mittel der Wahl in der Therapie der Pneumo- 
cystis carinii-Pneumonie bei AIDS-Patienten gilt, berichtete Th. Weinke, Berlin. Der Nachweis von Pneumocystis carinii sichert die Diagnose AIDS. Mehr als $80 \%$ der $\mathrm{Pa}$ tienten hatten irgendwelche Nebenwirkungen (gastrointestinale Beschwerden, Hautausschlag, Fieber, Leukopenie, Thrombopenie, Hepatose, Nierenveränderungen), die in den meisten Fällen jedoch geringeren Grades waren. Bei 10 von 59 Patienten war 4 mal durch großflächige Exantheme und $6 \mathrm{mal}$ durch Blutbildveränderungen bis zur Agranulocytose ein Therapieabbruch notwendig. 11 Patienten zeigten keinerlei Nebenwirkung. Von 7 Patienten mit einer allergischen Disposition gegen Antibiotika zeigten 6 deutliche Nebenwirkungen. Eine Leukopenie tritt meist zwischen dem 9. und 13. Behandlungstag auf. Der Autor empfahl deshalb, eine vorherige Sensibilisierung mit Sulfonamiden zu vermeiden und anschließend als Prophylaxe Fansidar zu geben. Außerdem solle $2 \mathrm{mal}$ wöchentlich eine Laborkontrolle erfolgen.

Einen kasuistischen Beitrag lieferte $D$. Nothdurft, München, der Anfang dieses Jahres 2 Fälle von Schlafkrankheit als importierte Erkrankung beobachtete. Bei beiden Patienten, die bei einem $1 \frac{1}{2}$ tägigen Aufenthalt in einem Nationalpark in Rwanda mehrfach von Tse-Tse-Fliegen gestochen wurden, bildete sich innerhalb weniger Tage eine schmerzhafte Primärläsion am Unterschenkel, denen am 7. bzw. 8. Tag Fieber folgte. Während ein käuflich erhältlicher IHA-Test negativ war, fiel ein selbst hergestellter Immunfluoreszenztest positiv aus. Im Serum war IgM erhöht. Im Liquor fand sich keine Reaktion. Da die Schlafkrankheit von 1976 von 5000 beobachteten Fällen auf 12000 Fälle im Jahr 1983 zugenommen hat, sollte beim Aufenthalt in Endemiegebieten an diese Erkrankung gedacht werden, die ohne Therapie tödlich verläuft.

Th. Löscher, München, berichtete über die Behandlung der Schlafkrankheit, die im hämatologischen Stadium mit Suramin (Germanin und Pentamidin [Lomidine]) und bei ZNS-Befall mit DFMO (Defluormethylornithin = Iflornithin), das Arsenpräparaten vorzuziehen ist, erfolgen sollte.

Einen weiteren kasuistischen Beitrag lieferte $H$. Sigl, München, über einen Patienten, der zur Notfalloperation wegen einer Gallenblasenerkrankung kam und bei dem der Befall mit dem großen Leberegel, der auch in Mitteleuropa vorkommt, nachgewiesen wurde. Die Diagnose sollte durch Laparotomie histologisch gestellt werden. Als Überträger kommen Salat, Sauerampfer, Kresse u.a. in Frage.

Einen kurzen Überblick über die infektionsepidemiologische Situation in der Bundesrepublik Deutschland 1987 gab H. Ph. Pöhn, Bad Nauheim. Von den 4 häufigsten Erkrankungen ist die Enteritis infectiosa (62377), davon $2 / 3$ Salmonellosen (39333) 1987 weiterhin angestiegen. Die Tuberkulose von der erst die Zahlen von 1986 vorliegen (14976) zeigt gegenüber den Vorjahren einen Rückgang, ebenso wie die Virushepatitis (11311) und die Meningitis/Encephalitis (3411). Die Malaria, die meist aus Afrika eingeschleppt wurde und 1986 stark angestiegen war, zeigt 1987 wieder einen leichten Rückgang. Bei den 3 gemeldeten Poliomyelitisfällen handelt es sich um einen Fall aus Südindien und zweimal um Impfschäden.

Der letzte Halbtag war den typischen und ,atypischen" Mykobakterien (Schwerpunkt AIDS), einem wie Mauch meinte, bisher stiefmütterlich behandelten Thema, gewidmet. Die Tuberkulose, die in den USA bis 1985 deutlich abnehmend war, zeigte 1986 und 1987 wieder einen leichten Anstieg, der wahrscheinlich durch das häufige Auftreten als opportunistische Erkrankung bei AIDS mitbedingt ist.

In einem kurzen Beitrag berichtete H.-G. Weber, Hannover, daß von nicht BCG-geimpften Schülern der berufsbildenden Schulen in Hannover über $40 \%$ einen positiven Tuberkulintest mit GT 10 aufwiesen, und daß auch die TBC-Erkrankungen gegenüber 1986 eine Zunahme zeigten.

Die Tuberkulose ist den Frauenärzten nicht bekannt, „die sehen wir nicht", sagte E. R. Weissenbacher, München, zum Thema tuberkulöse Infektionen bei gynäkologischgeburtshilflichen Patientinnen. Bei der Durchsicht von ca. 10000 Krankenblättern aus den Jahren 1976-1986 fand er nur 10 Genitaltuberkulosen. Die Diagnose wurde am häufigsten laparoskopisch gestellt. Untersucht werden sollte mittels Adapter gewonnenes Menstrualblut, das Abradat, OP-Material oder das .Douglas-Punktat. Befallen sind am häufigsten die Tuben (90-100\%), gefolgt vom Uterus $(50-60 \%)$ und den Ovarien $(20-30 \%)$. Klinisch finden sich in $10-40 \%$ Blutungsstörungen bis zur Amenorrhoe. Die Hälfte der Patientinnen haben uncharakteristische abdominale Beschwerden. Bei ca. $25 \%$ der Genitaltuberkulosen besteht Sterilität oder anders gesagt, $5-10 \%$ der sterilen Frauen haben eine Genitaltuberkulose.

Über zelluläre Grundlagen der Mykobakterieninfektionen bei Immuninsuffizienz sprach S. H. E. Kaufmann, UIm. Er stellte die Doppelfunktion der Makrophagen heraus, die einmal den wichtigsten Lebensraum für die Mykobakterien bilden, auf der Oberfläche mit mykobakteriellen Antigenen besetzt sind und so von T-Lymphozyten erkannt werden, die mit den infizierten Makrophagen interagieren, weitere Lymphozyten anlocken und zur Granulombildung. führen. Andererseits befindet sich der Makrophage im Kampf mit dem Mykobakterium, kann das Wachstum hemmen, aber die virulenten Mykobakterien nicht abtöten. Das Ganze befindet sich in einer Balance. Bei der Immuninsuffizienz kann es einmal zu einer Aktivierung latenter Infektionen (meist Mycobacterium tuberculosis) oder zu einer Neuinfektion mit atypischen Mykobakterien kommen. AIDS als Makrophagen-Krankheit macht den Organismus anfälliger gegen Mykobakterien.

Als wichtigste diagnostische Methode zur Erkennung von Mykobakterioseni bei AIDS sieht $H$. Mauch, Berlin, das mikroskopische Präparat an. So können positive Blutkulturen zum Teil auch schon im Ziehl-Neelsen-Präparat erkannt werden. An zweiter Stelle steht der kulturelle Nachweis, wobei Festkulturen und Flüssigkulturen, auch als radiometrische Verfahren (Bactec) angewendet werden können. Der Vorteil des radiometrischen Verfahrens, dessen positive Ausbeute zwar nicht größer als die mit konventionellen Kulturen ist, ist jedoch das schnellere Ergebnis. Demgegenüber stehen höhere Kosten und das Nichterkennen ubiquitärer Mykobakterien, die als „atypische" Mykobakterien eingeordnet werden. Weitere diagnostische Maßnahmen sind die Tuberkulinreaktion und serologische Methoden, die keine größere Bedeutung haben. Dazu kommen als neue diagnostische Verfahren der spezifische RNS-Nachweis (Genprobe) und der direkte Nachweis mit monoklonalen Antikörpern.

B. Ruf, Berlin, stellte die Klinik der Mykobakteriosen bei AIDS vor. Während sich bei HIV-negativen Patienten vorwiegend eine Erkrankung der Lungen findet, kommt es bei den HIV-positiven meist zu einer Dissemination mit einer Zunahme der atypischen Mykobakteriosen, bei denen Mycobacterium avium intracellulare weitaus an dèr 
Spitze steht. Es kommen auch Mycobacterium kansasii und fortuitum vor, die sonst keine Bedeutung haben. Führende Symptome sind Gewichtsverlust und Fieber; die Laborwerte sind unspezifisch.

G. Jautzke, Berlin, der zur Pathologie der Mykobakterieninfektionen bei AIDS sprach, hatte in 12 von 50 AIDSFällen Mykobakterien nachgewiesen, davon in 7 Fällen Mycobacterium avium intracellulare und in 5 Fällen Mycobacterium tuberculosis. Typisch für die Mycobacterium avium intracellulare-Infektion bei AIDS ist die nur dort auftretende mykobakterielle Histiocytose ohne Nekrosen mit ungehemmtem Wachstum in den Histiozyten (histiozytäre "Schaumzellen“). Daneben finden sich normale Tuberkel auch mit Verkäsung und Nekrosen wie bei Mycobacterium tuberculosis.

Zu den Therapiemöglichkeiten bei Mycobacterium avium intracellulare-Infektionen sagte $E$. Holzer, München, daß eine einigermaßen sichere Behandlungsmöglichkeit nicht bestehe. Mit Amicacinen entfiebern die Patienten innerhalb weniger Tage, das Mittel verliert aber nach kurzer Zeit seine Wirksamkeit. Auch mit den auf Grund der Testung wirksamen Mitteln ist oft nichts zu erreichen. Als wirksam im Test erweisen sich Cycloserin und Prothionamid. Ein Patient wurde mit kombinierter Behandlung von Clofazimine, Daxon und Cycloserin mykobakterienfrei. Holzer glaubt deshalb jetzt, diese Therapie möglichst frühzeitig einsetzen zu sollen.

H. Kern, Ulm, berichtete über den Fall einer Erkrankung an Mycobacterium marinae, einem im Veterinärbereich seit 1926 bekannten Erreger, der zu einer lokalen Erkrankung führt und als Schwimmbadgranulom oder Fischzüchtergranulom, in Japan seit 1984 als Berufskrankheit, bezeichnet wird. In Deutschland sind von 1963 bis 1987 25 Fälle beobachtet worden. Zur Diagnose führte das klinische Bild, die Expositionen in der Anamnese sowie Histologie und Kultur. Wichtig ist es zu beachten, daß das Temperaturoptimum $30^{\circ} \mathrm{C}$ beträgt, und daß das Mycobacterium marinae bei $37^{\circ} \mathrm{C}$ nicht wächst. Die Therapie sollte primär konservativ mit Rifampicin, Ethambutol und Doxycyclin erfolgen.

W. Hauck

\section{Mitteilungen}

\section{Neufassung der GOÄ}

Die geänderte Gebührenordnung für Ärzte - GOÄ ist am 1. Juli 1988 in Kraft getreten. Sie wurde veröffentlicht im Bundesgesetzblatt Teil 1, Nr. 26 vom 23. 6. 1988, Seite$797 \mathrm{ff}$.

\section{Postsendungen mit medizinischem Untersuchungsgut}

Wie das Bundespostministerium in Fernschreiben an die Oberpostdirektionen mitgeteilt hat, soll zu der Verfügung 257/88 (siehe Juniheft der Laboratoriumsmedizin, Seite BDL 70-71) eine ergänzende Amtsblattverfügung ergehen. In dieser Ergänzungsverfügung sollen die Übergangsfrist für die Verwendung bisher zugelassener Verpackungen bis zum 30.6. 1989 verlängert und der Versand als Warensendung auslaufend bis zum 30.9. 1988 toleriert werden. Dies gilt jedoch nicht für Versandgut, das für Mensch oder Tier infektiös ist oder bei dem ein entsprechend begründeter Verdacht gegeben ist.

Es ist vorerst davon auszugehen, daß sich der Geltungsbereich der Amtsblattverfügung 257/88 auf flüssiges Transportgut beschränkt (damit sind z. B. Abstriche oder Ausstriche auf Objektträgern ausgenommen) und daß für in Formalin fixierte Proben, bei denen jegliches Infektionsrisiko ausgeschlossen ist, Erleichterungen für den Versand vorgesehen sind (keine aufsaugenden Stoffe, bei großvolumigen Proben nur ein Gefäß erforderlich).

\section{Kostensenkung \\ im medizinischen Laboratorium durch Einsatz von EDV-Programmen}

Unter diesem Titel findet im Rahmen der Therapiewoche in Karlsruhe am Montag, dem 29. August 1988, ein Seminar statt. Die Leitung hat Dr. H. Wüst, Direktor des Medizinisch-Diagnostischen Instituts des Klinikums Karlsruhe. Themen: Einführung und Zielsetzungen der Kosten-Leistungsrechnung / Das Leistungsgerüst als Basis der Kosten-Leistungsrechnung / Kostenarten und Kostenstellenrechnung / Personalplanung und Personalkostenrechnung durch Strukturanalyse / Kostenträgerrechnung und Budgetierung gemäß BPfV / Maßnahmen zur Kosteneinsparung.

Wegen begrenzter Teilnehmerzahl wird um Anmeldung gebeten an das Sekretariat Dr. H. Wüst, Städt. Klinikum, Med.-Diagn. Institut, Moltkestr. 14, 7500 Karlsruhe 1, Tel. $0721 / 597861$.

Für Interessenten besteht die Möglichkeit, am nächsten Vormittag im Med.-Diagn. Institut an einer Demonstration mit praktischen Übungen teilzunehmen.

\section{AIDS-Forschungspreis der Deutschen Gesellschaft für Infektiologie}

Die Deutsche Gesellschaft für Infektiologie hat einen mit 10000, - DM dotierten „AIDS-Forschungspreis" ausgeschrieben, der jährlich vergeben werden soll. Für den ersten Preis, der während des 2. Deutschen AIDS-Kongresses, der vom 22. bis 24. Januar 1989 in Berlin stattfindet, verliehen wird, können Arbeiten bis zum 30. September 1988 unter einem Kennwort in 7facher Ausfertigung bei der Geschäftsstelle der Deutschen Gesellschaft für Infektiologie, Prof. Dr. med. E. Holzer, Städt. Krankenhaus Schwabing, IV. Medizinischë Abteilung, Kölner Platz 1, 8000 München 40, eingereicht werden.

\section{Erratum}

Vorschläge zum diagnostischen Vorgehen bei endokrinologischen Erkrankungen

Im Juniheft muß es auf Seite BDL 67 im Kapitel ,Nebennierenmark' unter ,Nachweisdiagnostik' richtig heißen: „meist mit Clonidin-Test: $\mathbf{0 , 3} \mathbf{~ m g}$ oral”. Dies entspricht einer Tablette Clonidin 300 Riker oder Clonidin-ratiopharm 300.

Wir bitten, das Versehen zu entschuldigen. 


\section{Gerhard-Domagk-Preis}

Der Gerhard-Domagk-Preis für klinische oder experimentelle Krebsforschung wird erneut ausgeschrieben. Ein Merkblatt mit den genauen Bedingungen des Kuratoriums der Stiftung Krebsforschung Professor Dr. Gerhard Domagk an der Westfälischen Wilhelms-Universität Münster kann bei Professor Dr. W. H. Hauss, em. Direktor der Medizinischen Universitätsklinik und Poliklinik, Domagkstr. 3. 4400 Münster, angefordert werden. Einsendeschluß für Bewerbungsarbeiten ist der 31. Dezember 1988.

\section{Robert-Koch-Preis 1988}

Auf Empfehlung des Wissenschaftlichen Beirats hat der Vorstand der Robert-Koch-Stiftung e.V. in seiner Sitzung am 15. Mai 1988 beschlossen, den Robert-Koch-Preis des Jahres 1988 Professor Dr. Donald Metcalf, Victoria/ Australien, zu verleihen. Die Robert-Koch-Medaille in Gold ist Dr. Willy Burgdorfer, Hamilton/USA zugesprochen worden.

Die feierliche Preisverleihung wird am Montag, 7. November 1988, in der Universität Bonn stattfinden.

\section{Personalien}

Dr. rer. nat. Gerd Döring, Tübingen, Privatdozent für Exp. Hygiene und Medizinische Mikrobiologie wurde zum C2-Professor ernannt.

Prof. Dr. Rolf Zinkernagel, Zürich, wurde mit dem OttoNaegeli-Preis 1988 für seine ,hervorragenden Leistungen auf dem Gebiet der Immunologie und der Krankheiten, die mit der Immunabwehr zusammenhängen", ausgezeichnet.

Prof. Dr. H.-M. Seitz, Direktor des Instituts für Medizinische Parasitologie, Bonn, wurde zum Prodekan der Medizinischen Fakultät gewählt.

Prof. Dr. W. Henkel, Direktor des Instituts für Medizinische Mikrobiologie der Medizinischen Universität Lübeck, ist in den wissenschaftlichen Beirat des RobertKoch-Instituts am Bundesgesundheitsamt berufen worden.

Dr. phil. Wolfgang Woloszczuk, wissenschaftlicher Mitarbeiter am Ludwig-Boltzmann-Institut für klinische Endokrinologie in Wien, erhielt die Lehrbefugnis als Universitätsdozent für Medizinische Chemie an der Medizinischen Fakultät der Universität Wien.

Dr. rer. nat. N. Müller-Lantzsch, Professor für Virologie in Freiburg, hat einen Ruf auf die C3-Professur seines Fachgebietes an der Universität des Saarlandes erhalten.

Prof: Dr. V. Sachs, Kiel, Direkter der Abteilung Transfusionsmedizin am Universitätsklinikum, wurde mit Ablauf des Monats März 1988 emeritiert.

Dr. M. Beato, Professor für physiologische Chemie in Marburg, hat den Ruf auf die C4-Professur für Molekularbiologie an der Universität angenommen.

Dr. H. E. Krampitz, München, Professor für vergleichende Tropenmedizin, vormals Abteilung für Infektionsund Tropenmedizin der Medizinischen Universitätsklinik Innenstadt, wurde von der Deutschen Gesellschaft für Parasitologie zum Ehrenimitglied gewählt.
Für die MTA/MTL

\section{Fortbildungsveranstaltungen der Landesgruppe NRW-Nord im dvta}

Die Landesgruppe NRW-Nord im dvta kündigt folgende Fortbildungsveranstaltungen an, die alle in den Schulen der Städtischen Kliniken, Kalkweg, 4100 Duisburg, stattfinden:

\section{August 1988}

$9.00-13.00$ Uhr

\section{Hämatologie}

1. Einführung in die Zellbiologie

2. Morphologische Kriterien-zur Beurteilung einer Zelle

3. Die normale Myelopoese

4. Pathologie der Myelopoese

\section{September 1988} $9.00-17.00 \mathrm{Uhr}$

\section{Elektronische Datenverarbeitung}

Zwei Themen stehen zur Auswahl und werden nach Teilnehmerinteresse veranstaltet.

1. Möglichkeiten der Programmierung in BASIC

2. Einführung in Datenbanksysteme

\section{Oktober 1988}

$9.00-13.00 \mathrm{Uhr}$

\section{Thrombocyten}

1. Thrombocytenfunktionsdiagnostik - ein vernachlässigter Aspekt im hämostaseologischen Labor

2. Morphologie und Biochemie der Thrombocyten

3. Diagnostik von Thrombopathien

4. Therapie von Thrombopenien und Thrombopathien

Anmeldung, Programm und Auskunft zu allen Veranstaltungen: Frau Regina Massalsky, Hausmannfeld 22, 4200 Oberhausen 1.

\section{Aus Österreich}

\section{Jahrestagung der Österreichischen Gesellschaft für Laboratoriumsmedizin 1988}

unter der Mitarbeit der Deutschen Gesellschaft für Laboratoriumsmedizin, im Kurzentrum Bad Ischl vom 8. 11. 6. 1988.

Das Ziel der heurigen Jahrestagung der ÖGLM war von Beginn an, mit Ideen, mit verstärkter Anwesenheit, in diesem Falle in Zusammenarbeit mit der DGLM unter der Leitung von Prof. Dr. L. Thomas als Kontaktperson, eine Demonstration einer permanent aufsteigenden, teils kämpfenden Gesellschaft; wie es die Gesellschaften für Laboratoriumsmedizin darstellen, zu definieren.

Als Ort wurde die kleine Stadt Bad Ischl im Salzkammergut mit einem funktionstüchtigen Kongreßzentrum gewählt, um in schöner Umgebung, in einer typisch österreichischen, legendären Stadt, ein familiäres Treffen zu gestalten. Daß dies gelungen ist, beweist die rege Teilnahme an dieser Tagung, 95 österreichische und 107 deutsche Kollegen, sowie 29 Vertreter der Industrie waren anwesend. 
Die Organisation der Jahrestagung war in dankenswerter Weise unter der perfekten Leitung des Sekretärs der ÖGLM, Herrn Dr. H. W. Pilgerstorfer hervorragend und hinterläßt allen Teilnehmern ein unvergeßliches Erlebnis. Das wissenschaftliche Programm unter der Leitung von Prof. Dr. P. Bayer, Wilhelminenspital, Wien war überzeugend aufgebaut. Die wissenschaftliche Ebene, wie sie der Festvortrag mit dem Titel „Molekularer Aufbau des Cytoskelettes", vorgetragen von Prof. Dr. G. Wiche, Wien, aufzeigte, die angewandte, praxisbezogene Erfahrung. die zukunftsorientierte Wissenschaft, wie das Thema "DNA-Sonde in der Labordiagnostik" sie darstellte, und die Ebene der Standespolitik waren ein überzeugendes Kräfteparallelogramm dieser Jahrestagung 1988.

Ein wesentliches Merkmal dieses Meetings war von $\mathrm{Be}$ ginn an die Inkludierung der Industrie mit einem Vertreter der ÖDGH (Österreichische Gesellschaft der Diagnostika- und Gerätehersteller) bei der Organisation dieses Kongresses. Von beiden Seiten zeigte sich eine gute $\mathrm{Zu}$ sammenarbeit; die Eröffnung der Ausstellung am Vorabend des Tagungsbeginnes demonstrierte dies mit einem gutgelungenen "get together".

Die gut besuchten Symposien: Neuere Entwicklungen bei der Mechanisierung immunchemischer Verfahren, die Labordiagnostik kardiologischer Sekundärerkrankungen waren von der Thematik her treffend gewählt.

Bewährt hat sich weiters, vor dem eigentlichen Kongreß einen Workshop abzuhalten. Es wurde im kleineren Kreis im Kurhotel Bad Ischl über das Gelenkspunktat erfolgreich diskutiert. Man weiß jetzt, was der behandelnde Arzt wirklich vom Laborarzt erwartet.

Auch die Vereinigungen für Qualitätssicherung, ÖQUASTA und INSTAND benützten die Gelegenheit, vor der Jahrestagung der ÖGLM sich mit einem kurzen Halbtagsprogram über den derzeitigen Stand der Qualitätskontrolle zu informieren und einen gemeinsamen Gedankenaustausch zu pflegen. Das standespolitische Forum als Abschiuß der Tagung war eine Pflicht und Notwendigkeit, den in Ausbildung stehenden Ärzten, wie den in der Praxis und in den Spitälern tätigen Kollegen gegenüber, Unsicherheiten, Anerkennung des Facharztes und die Situation in der Zukunft zu diskutieren. Hierbei war der Erfahrungsaustausch mit den deutschen Kollegen von großer Wichtigkeit und für beide Teile eine gute Information.

Was wäre eine Tagung ohne Gelegenheit, den menschlichen Dialog pflegen zu können? Dafür sorgte ein ausgewogenes gesellschaftliches Programm, mit kulturellem Niveau, mit heiterer Geselligkeit auf einer nahegelegenen Alm und einem festlichen Abschluß in der Kaiservilla Bad Ischl. Auch diese Veranstaltungen waren überaus gut be: sucht. Man konnte diese Jahrestagung als familiäres Meeting im wahrsten Sinn des Wortes bezeichnen. Bei der Jahreshauptversammlung der ÖGLM wurde ein neues Team gewählt, Prof. P. Bayer, Leiter des Zentrallabors des Wilhelminenspitals der Stadt Wien wird als Präsident in den nächsten zwei Jahren die Gesellschaft lenken und leiten. Im Namen aller Fachkollegen wünschen wir ihm viel Erfolg für dieses Amt und geben der Hoffnung Ausdruck bei der nächsten Jahrestagung 1989, die in Wien stattfinden wird, sich mit viel innovativen Kräften wiederzusehen.

Prim. Dr. W. Hohenwallner

$\mathrm{KH}$ der Barmherzigen Schwestern

Langgasse 16, A-4020 Linz

\section{Neues Präsidium der ÖGLM}

Auf ihrer Jahreshauptversammlung am 10.Juni 1988 in Bad Ischl wählte die Österreichische Gesellschaft für Laboratoriumsmedizin ein neues Präsidium für die Funktionsperiode 1988-1990, das sich wie folgt zusammensetzt:

Präsident:

1. Vizepräsident:

2. Vizepräsident:

Past-Präsident:

Sekretär:

Finanzreferent:

Weitere Mitglieder:

Doz. Dr. Endler (zuständig für Ausbildung und Fortbildung)

MR. Dr. Lackner (zuständig für Verbindung zur Österreichischen Ärztekammer)

Dr. Pilgerstorfer (zuständig für Qualitätssicherung)

Dr. Scholda (Sonderaufgaben)

Kooptiert und im Bedarfsfall zu Vorstandssitzungen beizuziehen:

MR. Dr. Mustafa, MR. Dr. Jauk, Prim. Dr. Sommer, Prim. Dr. Klein, Dr. Schwaninger, Dr. Holzer.

Rechnungsprüfer:

Dr. Unger, Prim. Dr. Weissmann.

\section{Greiner-Nachwuchspreis}

Bei der Jahrestagung der Österreichischen Gesellschaft für Laboratoriumsmedizin in Bad Ischl wurde am 9. Juni 1988 Herr Mag. Walter Motz, Wels, für seine Arbeit „Osteocalcin bei chronischen Haemodialyse-Patienten als zusätzlicher Parameter zur Diagnose des fortgeschrittenen sekundären Hyperparathyreodismus" mit dem Greiner-Nachwuchspreis in der Höhe von S10000,ausgezeichnet.

\section{Leserzuschriften}

Zum zweiten Bericht einer Arbeitsgruppe der Deutschen Vereinigung zur Bekämpfung der Viruskrankheiten e.V. "Beschäftigung Schwangerer in medizinischen Laboratorien", den wir im Februar-Heft 1988 (Lab.med. 12, BDL 9-12) abdruckten, erhielten wir folgende Zuschrift:

Der redaktionelle Text im Kasten entspricht nicht den Tatsachen. Ein Teil der Kommentare wurde nämlich nicht berücksichtigt; so z. B. meine Leserzuschrift in Lab.med. 9, BDL 88 (1985). Mindestens 20 schriftliche Anfragen an Herrn Maas mit der Bitte um Stellungnahme zu dieser Zuschrift wurden ausnahmslos dilatorisch ohne jedes Ergebnis beantwortetet.

Ich hatte um Beantwortung folgender Fragen gebeten:

1. Welches Zahlenimaterial über. Erkrankungen Schwangerer in medizinischen Laboratorien lag Ihnen für die Bundesrepublik vor Verabschiedung dieser Richtlinien vor?

Lab.med. 12, Nr. 7: BDL 87 (1988)

$B D L 87$ 
2. Woraus schließen Sie, daß schwangere Frauen in medizinischen Laboratorien an Infektionskrankheiten häufiger erkranken als nicht schwangere Frauen?

3. Welche Beweise stehen Ihnen zur Verfügung für die Annahme, daß bei strikter Einhaltung der von Ihnen verabschiedeten Richtlinien eine signifikante Reduktion von Infektionen Schwangerer in medizinischen Laboratorien eintreten wird?

Dieselben Fragen stelle ich heute erneut, drei Jahre später.

Die BG Gesundheitsdienst und Wohlfahrtspflege teilt mir mit Schreiben vom 24. 3. 1988 lakonisch mit:

„Zu unserem Bedauern müssen wir Ihnen mitteilen, daß seitens der BGW keine Statistik über anerkannte Berufskrankheiten (Infektionskrankheiten) bei Schwangeren geführt werden, die in medizinischen Einrichtungen beschäftigt waren." Gez. Steffen

Im Virusreport der DVV vom 24. 1. 1985 (Autor unter anderen Prof. Dr. G. Maass) wird berichtet:

„Durch sachlich unberechtigte Berichte zur Gefährdung von Personen und Patienten in Krankenhäusern durch das mit AIDS assoziierte HTLV-3-Virus könnte der falsche Eindruck eines erhöhten Infektionsrisikos mit HTLV 3 in diesem Bereiche entstehen."

Interessant sind in diesem Zusammenhang auch die Diskussionen über das Beschäftigungsverbot schwangerer Anästhesistinnen während der gesamten Schwangerschaft, das von einigen Gewerbeärzten und Gewerkschafterinnen gefordert wird; nachzulesen in der ÄrzteZeitung vom 24. 11. 1987 auf Seite 21. Anästhesistinnen fühlen sich nämlich in ihrem beruflichen Fortkommen behindert, wenn es soweit kommen sollte und wenden u.a. ein, in jeder Schlagsahne-Spraydose sei pures Lachgas, aber niemand habe die Lachgaskonzentration im Blute Schlagsahneessender Schwangerer gemessen.

Es fehlt wieder einmal eine solide Datenbasis für die "Richtlinien”.

Wetten, daß der höfisch-universitäre Stil diverser Kollegen ihnen verbietet, die gestellten Fragen zu beantworten?

Dr. med. Dipl. biochem. Rudolf H. Seuffer

Ferdinand-Lassalle-Str. 40

7410 Reutlingen 11

\section{Erwiderung auf den Leserbrief von Dr. R. H. Seuffer:}

Der Leserbrief von Dr. R. H. Seuffer kann als Beispiel für einige Zuschriften gelten, die wir bereits nach Veröffentlichung unseres ersten Berichtes erhalten hatten; auf diese Zuschriften waren wir in dem zweiten Bericht absichtlich nicht eingegangen. Seine Abneigung gegen den „höfisch-universitären Stil" hindert Dr. Seuffer offenbar daran, den veröffentlichten Text zu lesen, sonst wäre ihm folgender Satz aufgefallen: „Der Nachweis der Gesundheitsschädigung beim Menschen kann in der Präventivmedizin auch sonst nicht generell als Voraussetzung schützenden Handelns verlangt werden." Unsere Absicht war, einen Überblick über die bestehenden Rechtsvorschriften und die aus ihnen abzuleitenden praktischen Schutzmaßnahmen bei der Beschäftigung Schwangerer in medizinischen Laboratorien zu geben; das haben die meisten Leser verstanden - einer wollte es offensichtlich nicht verstehen.

Prof. Dr. Maass (DVV)

Prof. Dr. Deinhardt (DVV)

Dr. Hoffmann (Vereinigung Staatl. Gewerbeärzte e.V.)

Prof. Dr. Norpoth (Deutsche Ges. f. Arbeitsmed. e.V.)

Dipl.-Ing. Thanner (Berufsgen. Gesundheitsd. u. Wohlfahrtspfl.)

\section{Aus dem DIN Deutsches Institut für Normung e. V.}

Der Normenausschuß Medizin (NAMed) im DIN Deutsches Institut für Normung e. V. legte im Mai 1988 folgende Normen vor:

DIN 58948, Teil 16

Sterilisation

Gas-Sterilisatoren

Betrieb von Formaldehyd-Gas-Sterilisatoren

Die Norm weicht in Einteilung und Einzelheiten von dem im Mai 1987 vorgelegten Entwurf ab.

So wurde der z.Zt. gültige MAK-Wert für Formaldehyd $(0,6 \mathrm{mg} /$ $\mathrm{m}^{3}$ ) aufgenommen und ein Abschnitt ,Auswahl des Sterilisiergutes" eingefügt.

DIN 58950, Teil 3

Sterilisation

Dampf-Sterilisatoren

für pharmazeutische Sterilisiergüter

Abnahmeprüfungen

Auch diese Norm weist geringfügige Abweichungen gegenüber dem Entwurf vom April 1987 auf.

Folgender Normentwurf wurde im Mai 1988 vorgelegt:

DIN 58946, Teil 3

Sterilisation

Dampf-Sterilisatoren

für medizinische Sterilisiergüter

Groß-Sterilisatoren / Prüfung auf Wirksamkeit

Dieser Normentwurf wurde vom Arbeitsausschuß „Dampf-Sterilisatoren" (Obmann: K. Scheel, Bad Schwartau) erstellt. Er soll die Ausgabe 11.81, deren Inhalt vollständig überarbeitet wurde, ersetzen.

Stellungnahmen werden bis 31: August 1988 an den Normenausschuß Medizin (NAMed) im DIN Deutsches Institut für Normung e. V., Postfach 1107, 1000 Berlin 30, erbeten.

Im Juni 1988 wurde nachstehende Norm vorgelegt:

DIN 58948, Teil 1 A1

Sterilisation

Gas-Sterilisatoren

Begriffe

Diese Norm ersetzt die Ausgabe 12. 86. Sie wurde um die im Entwurf vom September 1987 aufgeführten Begriffe erweitert.

\section{Zitát}

„Man kann eine Schwangerschaft nicht unterbrechen, sondern nur abbrechen. Man sollte deshalb nicht von Interruptio, sondern von Abruptio sprechen." (E. R. Weisenbacher, München, auf dem 14. Symposium der Deutschen Gesellschaft für Infektiologie 1988). 


\section{Buchbesprechungen}

\section{Handkommentar BMÄ, E-GO und GOÄ}

Von $\boldsymbol{H}$. Wezel und $\boldsymbol{R}$. Liebold. 4. Lieferung der 6. Auflage, Stand 1. 4. 1988. 482 Seriten, Laseblattsammlung. Asgard Verlag Dr. Wemer Hippe KG, St. Augustin. ISBN 3-537-53404-3. Preis des Gesamtwerkes (1342 Seiten) DM 98, -.

Bereits 3 Monate nach der letzten Lieferung erscheint eine umfangreiche Nachlieferung, die es erforderlich macht, etwa ein Drittel aller Blätter auszutauschen. Notwendig war dies, da vom Bewertungsausschuß zum 1. Januar 1988 beschlossene Änderungen und Ergänzungen, die nicht mehr fristgerecht zum Jahreswechsel eingearbeitet und kommentiert werden konnten, sowie weitere vertragliche Regelungen zum BMÄ, die ab 1. April gelten, aufzunehmen waren. Viele Anfragen zeigten Regelungslücken, noch bestehende Unklarheiten und Fehlmeinungen, die die Autoren zu weiteren Ergänzungen und Klarstellungen veranlaßten.

Auch die Ergānzung zeigt, daß es eine Reihe regionaler Besonderheiten gibt. So kann in Süd-Württemberg der Arzt (anstelle der EBM-Regelung, die sich nur auf Telefonate mit dem Krankenhaus erstreckt) die telefonische Unterrichtung eines anderen Arztes bei besonders gelagerten Krankheitsfällen anstelle eines Briefes ärzlichen Inhalts mit DM 5,- berechnen. Im Interesse des Kranken geführte Telefongespräche (z. B. dringende Krankenhauseinweisung. Vergiftungsfälle, dringende Befundmitteilung) können pro Telefonat mit DM 0,50 abgerechnet werden. Kompliziert wird die Abrechnung durch Sonderregelungen, wie z. B. in Süd-Württemberg, die nur für Betriebs- und Innungskrankenkassen, nicht aber für die AOK gelten (Nr.9002 Besuch durch eine Hilfskraft $=$ DM 6,-). Neu aufgenommen wurde zur Ziffer 319 der Zusatz, daß die Abstrichentnahme aus Bereichen desselben Organsystems nur einmal berechnungsfähig ist.

Mit Wirkung vom 1. Januar 1988 wurden im Hinblick auf die erheblichen Sachkosten bei arthroskopischen Leistungen die Punktzahlen bei den Nummern 2445-2450 deutlich, zum Teil über das Doppelte angehoben. Eine entsprechende Anpassung vermißt man noch bei den unterbewerteten labormedizinischen Leistungen. Eingefügt wurde eine Ubersicht über mögliche Kombinationen bei der Abrechnung von Bestandteilen des Blutbildes.

W. Hauck

\section{Laborfibel}

Hinweise und Anleitungen für den Anfänger im chemischen Laboratorium von $H$. Kruse. X, 166 S., 32 Abb., 7 Tab., brosch. VCH Verlagsgesellschaft mbH, Weinheim, 1988. ISBN 3-527-267662. DM 34, -

Ausnahmsweise eingangs eine subjektive Aussage: der Referent gesteht, sich nach der Lektüre dieser Laborfibel in sie verliebt und umgehend erneut darin geschmökert zu haben. - Zielgruppe dieser Fibel sind alle, die eine Ausbildung als Chemielaborant,
Chemotechniker. Chemisch-technischer Assistent aufnehmen wollen oder auch als Medizinisch-. Pharmazeutisch- oder Biologisch-technischer Assistent (-innen) sich für Chemie interessieren. .Das Buch soll dem Anfänger im chemischen Labor als Lernhilfe dienen. ihm grundlegende Kenntnisse der praktischen Arbeit vermitteln und ihm somit den Einstieg in die chemische Laboratoriumspraxis erleichtern" (aus dem Vorwort). Es soll? Nein, es wird. Man spürt auf jeder Seite das handfeste Wissen des Autors um die Nöte und das Interesse seiner Ansprechpartner. Er beginnt gottlob gleich mit Wichtigem für den Anfänger: Si cherheit im Labor (Gefahrenquellen durch Strom. Feuer. Git). erste Hilfe und Brandbekämpfung. Er fährt fort mit allgemeinen Hinweisen zum praktischen Arbeiten, Methodik, Protokollierung. Umgang mit Chemikalien und Gerāten sowie Reinigung. Im Hauptteil kommt er dann zur Sache und führt den begierig wartenden jungen Menschen ein in die Künste des Erhitzens, Kühlens, Filtrierens, Zentrifugierens, Wägens, des Zerkleinerns, Mischens und Trocknens, der Messung von Volumina. Temperaturen und pH-Werten. Man sieht den Autor geradezu in der Rolle eines begeisterten Chemikers, der seinen Sohn in diese faszinierende Welt der Chemie einführt. Und doch ist der Text exakt. konzentriert sich auf das Herz einer Beschreibung und verliert nie den fachlichen Anspruch. Das ist kein populärwissenschaftliches Getue, kein Comicheft für Möchtegernalchimisten (trotz lustiger Cartoons). Ein Hundsfott, der sich an manchem echt stört: man .eicht" nicht, sondern kalibriert (S.89): man bräuchte nicht unbedingt nur 3 Photos, die dann auch noch nur $3 \mathrm{pH}$-Meter zeigen: man könnte auf die Anrede in der zweiten Person verzichten. Aber was solls: der Referent, ein in Jahrzehnten in Industrie und Klinik ergrauter Chemiker, hat die Fibel zu seinen Handbüchern gestellt und eine zweite fürs Labor gekauft.

Walter Appel, Karlsruhe

\section{Eingegangene Bücher}

Gebührenordnung für Ärzte - GOÄ. Von Manfred Lieber. Textausgabe nach dem Stand vom 1. Juli 1988. Ca. 200 Seiten. kartoniert. Bundesanzeiger Verlagsges. mbH, Köln, 1988. ISBN 3-88784-157-3. DM 16,50.

Gesundheitsberatung durch Ärte. Ergebnis eines Modellversuchs in Hamburg und in der Pfalz. Hrsg. vom Zentralinstitut für die Kassenärztliche Versorgung in der Bundesrepublik Deutschland. Gesamtbearbeitung J. Bengel, U. Koch und Ch. Brühne-Scharlau. Wissenschaftliche Reihe - Band 32. 551 Seiten mit zahlreichen Tab., broschiert. Deutscher ArzteVerlag. Köln. 1988. ISBN 3-7691-8031-3.

Das Magnesiummangelsyndrom. Bedeutung für Mensch, Tier und Pflanze. Von H.-J. Holtmeier. 206 Seiten, 34 Abb., 54 Tab., broschiert. Hippokrates Verlag. Stuttgart, 1988. ISBN 3-7773-0846-3.

Parat. Jahrbuch Chemielabor 1988. Hrsg. v. S. Ebel und W. Dorner. 138 Seiten, zahlreiche Abb. und Tab., gebunden. $\mathrm{VCH}$

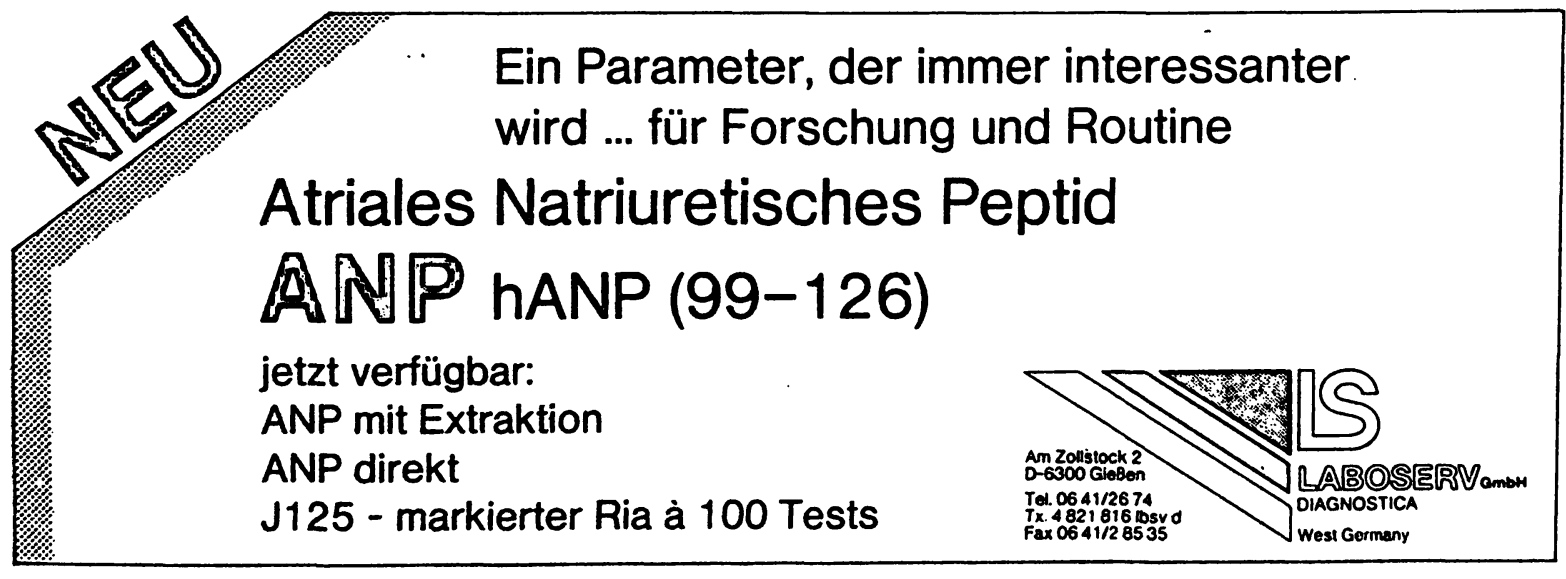


Verlagsgesellschaft, Weinheim, 1988. ISBN 3-527-26601-1. DM 40. - .

Physikalisch-chemische Methoden im klinischen Laboratorium. Von R. Franke / K. Thiele / F. Hofmann. Anleitungen für klinische Laboratoriumsmethoden, Band II. 3., überarbeitete Auflage. 220 Seiten, 101 Abb., 25 Tab., gebunden. VEB Verlag Volk und Gesundheit, Berlin, 1988. ISBN 3-333-00165-9. DM 28. - .

Infektionen und Impfungen in der Schwangerschaft. Infektionen der Mutter und des Feten - Schutzimplungen in der Schwangerschaft. Von Gisela Enders. X, 292 Seiten, $20 \mathrm{Abb}$. 38 Tab., 9 Schemata, gebunden. Urban \& Schwarzenberg, München, Wien, Baltimore, 1988. ISBN 3-541-11811-3. DM 58,-

Mikrobiologische Diagnostik in der ärztlichen Praxis. Ein Leitfaden. Von R. Gallien. VI, 155 Seiten, 147 Abb., 41 Tab., gebunden. Gustav Fischer Verlag, Stuttgart, New York, 1988. ISBN 3-437-11116-7. DM 68, - .

Lehrbuch der Medizinischen Mikrobiologie. Begründet von $H$. Reploh und H.-J. Otte. Hrsg. von Henning Brandis und Gerhard Pulverer. 6., neubearbeitete Aufl. XXIV, 759 Seiten, 236 Abb., 167 Tab., 4 Farbtafeln, kartoniert und gebunden. Gustav Fischer Verlag, Stuttgart, New York, 1988. Kart. ISBN 3-437-00484-0. DM 72.-. Geb. ISBN 3-437-00483-2. DM 89, - .

Trends in der Hämatologie. Tagungsbericht der Linzer Laborrunde, Bad Gastein, 28. 2. und 1. 3. 1985. Hrsg. von W. Hohenwallner, Redaktion: A. J. Born. 176 Seiten, zahlreiche Tab. und Abb., kartoniert. Veröffentlicht von TOA MEDICAL ELECTRONICS Deutschland GmbH, Hamburg, 1987. Schutzgebühr DM 58, - .

SIGNATUR Nr. 8 ,Album d'un amateur". Von Claude Simon. Text/Bild-Collage. 56 Seiten plus Übersetzungsbeilage. Auflage: 990, numeriert, handsigniert. Verlag Rommerskirchen, Remagen-Rolandseck, 1988. ISBN 3-926943-07-6. Im Abonnement DM 185, - inkl. Acrylglasrahmen.

\section{Kurzzitate}

Von 1000 untersuchten Tropenrückkehrern hatten 36 (=3,6\%) Entamoeba histolytica. Von den 21 Fällen, in denen eine Isoenzymdifferenzierung mittels Stärkegel-Elektrophorese gelang, zeigten 4 ein pathogenes Isoenzymmuster (Zymodem), was mit der klinischen Symptomatik und positiven Serologie korrelierte. [Dtsch. med. Wschr. 113, 678-682 (1988).]

Da Infektionen mit Hepatitis-D-Virus häufiger sind als bisher angenommen (Durchseuchungsrate bei Probanden aus normaler Bevölkerung mit akuter HBV-Infektion ca. $2 \%$, mit chronischer HBV-Infektion ca. $11 \%$ ), sollte in den Umfang der routinemäßigen serodiagnostischen Untersuchungen bei Hepatitis auch die Untersuchung auf HDV-Marker aufgenommen werden. [Bundesgesundhbl. 31, 129-133 (1988).]

Der in vitro-Nachweis der Bildung von HIV-spezifischem IgG im Überstand der Kultur peripherer Lymphozyten kann in der Frühdiagnose von HIV-Infektionen bei Kindern hilfreich șein, da von der Mutter übertragene Antikörper bis zu 15 Monaten persistieren. (Lancet 1988/I, 852-854.)

Ein 22jähriger Mann erkrankte am Tag vor der Rückreise nach einem 3wöchigen Urlaub ohne Malariaprophylaxe in Zaire mit hohem Fieber und Schwellungen im Halsbereich sowie einer erbsgroßen druckschmerzhaften Schwellung am behaarten Kopf (Trypanosomenschanker). Malariaerreger wurde nicht gefunden, aber extrazelluläre Trypanosomen (akute Schlafkrankheit). Antikörper waren erst nach 3 Monaten nachweisbar. [medwelt 39, 505-506 (1988).]

Mit Hilfe der indirekten Immunfluoreszenz konnten an frischen polymorphkernigen Granulozyten antizytoplasmatische Antikörper (ACPA; Synonym: ANCA = anti-neutrophil cytoplasmic antibodies) bei $73 \%(77 / 105)$ der Patienten mit Wegenerscher Granulomatose nachgewiesen werden, dagegen nur bei 6 von 1500 Kontrollpersonen $(<1 \%)$. Bei aktiver Wegenerscher Granulomatose waren sie stets nachweisbar, bei einer Exazerba- tion stieg der Titer an. [Dtsch. med. Wschr. 113, 413-417 (1988).]

Bei dem seltenen, in der Bundesrepublik Deutschland erst sechsmal beschriebenen, Churg-Strauss-Syndrom (nekrotisierende systemische Vaskulitis mit Asthma bronchiale und periphe. rer Eosinophile) findet sich neben der Eosinophile. die im allgemeinen zwischen $1,5 \times 10^{9}$ und $5 \times 10^{9}$ Zellen pro Liter und damit zwischen den Werten beim allergischen Asthma bronchiale und dem hypereosinophilen Syndrom liegt, labordiagnostisch lediglich eine IgE-Erhöhung in $75 \%$ der Fälle. Unspezifische Entzündungsparameter (CRP, RF, C3, C4, IgG, BSG) sind gelegentlich erhöht. [Med. Klin. 83, 223-227 (1988).]

Bei ungeklärter Genese einer Diarrhoe/Malabsorption sollte eine Giardiasis (Giardia lamblia) durch Stuhluntersuchungen ausgeschlossen werden. Zur Behandlung stehen Nitroimidazol-Präparate zur Verfügung. Wegen Rezidivneigung sollten Kontrolluntersuchungen bis 7 Wochen nach der Behandlung durchgeführt werden. [Med. Klin. 83, 219-222 (1988).]

Die pseudomembranöse Kolitis ist heute meist Folge einer Antibiotikatherapie, bei der die normale Darmflora durch den anaeroben Sporenbildner Clostridium difficile überwuchert wird. Am häufigsten tritt sie nach Clindamycintherapie auf. Die Diagnose erfolgt durch. Keim- und/oder Toxinnachweis; therapeutisch wird Vancomycin angewendet. [Med. Klin. 83, 199-205 (1988).]

In 55\% der Fälle mit einer Pneumocystis carinii-Pneumonie konnte die Diagnose durch eine Sputumuntersuchung gestellt werden. Dabei war die Immunfluoreszenzfärbung mittels monoklonaler Antikörper empfindlicher (45 von 49 ) als die Toluidinblau-0-Färbung (39 von 49) und die Giemsafärbung (Diff-Quik) (37 von 49). [The New England Journal of Medicine 318, 589593 (1988).]

Der früher übliche Ausdruck „Alpha-1-Antitrypsin” ist zugunsten des allgemeinen Terminus ,Alpha-1-Pi" verlassen worden, da sich die antagonistische Wirkung weniger auf Trypsin als auf Elastasen bezieht. [Dtsch. Med. Wschr. 113, 369-373 (1988).]

Von 11588 innerhalb von 12 Monaten im Waldviertel (Niederösterreich) erfaßten Patienten hatten $74(0,64 \%)$ eine Hypercalcämie (Calcium > 10,5 mg/dl). 20 der 74 hypercalcämischen Patienten (27\%) hatten einen primären Hyperparathyreoidismus; von den restlichen Patienten mit normalem oder supprimiertem Parathormonspiegel hatten 15 Patienten (20\%) ein Malignom, 6 davon mit Knochenmetastasen. [Dtsch. med. Wschr. 113, 163-168 (1988).]

Die Kombination der Tumormarker NSE (neuronenspezifische Enolase), CEA (Karzinomembryonales Antigen) und SCC (Squamous Cell Carcinoma Antigen) erlaubt mit hoher Șicherheit die Unterscheidung zwischen benignen Lungenerkrankungen und kleinzelligen Bronchialkarzinomen. Dies beruht einerseits auf der hohen Sensitivität der NSE für kleinzellige Bronchialkarzinome, zum anderen darauf, daß pathologische SCC-Werte in diesen Fällen nicht vorkommen. [Med. Klin. 83, 125-128 (1988).]

Beim Vergleich eines Enzymimmunoassays (Chlamydiazyme) und eines direkten Immunfluoreszenztestes mit monoclonalen Antikörpern (MikroTrak) zur Chlamydiendiagnostik fiel die hohe Übereinstimmung der positiven Resultate auf, die mit der Kultur nicht bestätigt werden konnten. Von 112 Untersuchungen, in denen 1, 2 oder 3 der Methoden positiv ausfielen, war Chlamydiazyme positiv in 89 , MikroTrak in 90 und die Zellkultur in 48 Fällen. Davon fand sich eine Übereinstimmung bei allen 3 Methoden in 37 Fällen, bei den beiden immunologischen Methoden in ebenfalls 37 Fällen; Chlamydiazyme und Kultur zeigte in 2 Fällen, MikroTrak und Kultur in ebenfalls 2 Fällen Übereinstimmung. Nur eine positive Methode fand sich $13 \mathrm{mal}$ bei Clamydiazyme, $14 \mathrm{mal}$ bei MikroTirak und $7 \mathrm{mal}$ in der Zellkultur. [Schweiz. med. Wschr. 118, 223-226 (1988).]

Eine exzessive Hyperandrogenämie bei einer Patientin mit Leyd́dig-Zell-Tumor ging einher mit Hypercholesterinämie mit erhöhter $\beta$-Lipoprotein- und erniedrigter $\alpha$-Lipoproteinfraktion (Quotient $\beta$-Lipoprotein zu $\alpha$-Lipoprotein-Cholesterin $=3,0$ ). Nach Operation normalisierte sich die periphere Testosteronkonzentration bei deutlichem Absinken von $\beta$ - und Prä- $\beta$-Cholesterin, nach Östrogensubstitution stieg der $\alpha$-Cholesterinanteil deutlich an $(\beta / \alpha$-Lipoprotein-Quotient $=1,2)$. [Dtsch. męd. Wschr. 113, 218-220 (1988).] 


\section{Tagungen}

Ghent (Belgien): 23. bis 26. August 1988 - 7th International Symposium on Mass Spectrometry in Life Sciences.

Auskunft: Prof. Dr. A. De Leenheer, Laboratoria voor Medische Biochemie en voor Klinische Analyse, Rijksuniversiteit Gent, Haelbekestraat 72. B-9000 Ghent.

Tübingen: 29. August bis 2. September 1988 - Humane Monoklonale Antikörper: Herstellung und Anwendung.

Leitung: Prof. Dr. W. Bessler, Freiburg, und Priv.-Doz. Dr. D. Baron, Mannheim).

Auskunft: Universität Tübingen, Arbeitsstelle Wissenschaftliche Fort- und Weiterbildung. Wilhelmstr. 5, 7400 Tübingen, Tel.: 07071/29-6439 oder 29-5010.

Münster: 28. bis 29. September 1988 - Biochemistry of Macrophages. Symposium der Studiengruppe Molekulare Immunologie der Gesellschaft für Biologische Chemie.

Themen: The Mononuclear Phagocyte System / Colony-Stimulating Factors / Macrophage/Endothelial Cell Interactions / Lipoprotein Metabolism in Macrophages / Accessory and Effector Functions / Macrophages in Inflammation.

Auskunft: Frau B. Scheibel, Institut für Experimentelle Dermatologie, VonEsmarch-Str. 56, 4400 Münster, Tel.: 0251 / 836578.

Essen: 29. September bis 1. Oktober 1988 - Strahlenschutz-Grundkurs für Mediziner und Nichtmediziner (z. B. Medizinphysiker, Chemiker, Biologen, die im medizinischen Bereich tätig sind), gemäß der Röntgen- und Strahlenschutzverordnung über den Erwerb der Fachkunde im Strahlenschutz bei der Anwendung von Röntgenstrahlen, radioaktiven Stoffen und Bestrahlungsanlagen im medizinischen Bereich, entsprechend den neuen Richtlinien zu der Röntgenverordnung vom 8. Januar 1987 und der ".Richtlinie Strahlenschutz in der Medizin”, Anlage A3, Ziffer 1.1 und Anlage A3, Ziffer 2.1. Gemeinsames Ministerialblatt Nr.31, S.638, vom 10. Nov. 1979.

Auskunft: Haus der Technik e.V., Hollestr. 1, 4300 Essen 1, Tel.: 0201 / 1803-1.

Rom (Italien): 3. bis 7. Oktober 1988 - 3rd International Symposium on "Epstein-Barr Virus and Associated Malignant Diseases".

Themen: Viral proteins / Immunopathology / Humoral and cellular immunity / Oncogenes / Vaccines / Antiviral drugs / Immunotherapy / Genotypic and phenotypic aspects of virus-cell interaction/Etc.

Auskunft: Dr. Alberto Faggioni, Reparto di Medicina Sperimentale, Viale Regina Elena, 324, I-00161 Roma, Tel.: 06/494-0540/492791.

Essen: 6. bis 8. Oktober 1988 - Spezialkurs zum Erwèrb der Fachkunde im Strahlenschutz als ermächtigter Arzt - Teil I nach der Strahlenschutz-Gesetzgebung.

Auskunft: Haus der Technik e.V., Hollestr. 1, 4300 Essen 1, Tel.: 0201 / 1803-1.

Essen: 13. bis 15. Oktober 1988 - Strahlenschutz-Kurs für medizinisch-technische Assistenten und Radiologie-Assistenten.

Auskunft: Haus der Technik e.V., Hollestr. 1, 4300 Essen 1, Tel.: 0201 / $1803-1$.

Essen: 13. bis 15. Oktober 1988 - Strahlenschutz-Spezialkurs für Nuklearmediziner und Labormediziner über den Erwerb der Fachkunde im Strahlenschutz bei der Anwendung von offenen radioaktiven Stoffen im medizinischen Bereich, entsprechend der „Richtlinie Strahlenschutz in der Medizin". Anlage A3, Ziffer 1.2, Gemeinsames Ministerialblatt Nr.31, S.638, vom Nov. 1979.

Auskunft: Haus der Technik e.V., Hollestr. 1, 4300 Essen 1, Tel.: 0201 / 1803-1.

Erlangen: 21. und 22. Oktober 1988 - Arbeitstagung der Deutschen Vereinigung zur Bekämpfung der Viruskrankheiten e.V. (DVV): Molekularbiologie in der virologischen Diagnostik.

Themen: Anwendungsmöglichkeiten und Methoden der molekularen Virusdiagnostik / Herpesviren / Hepatitis / Parvoviren / Papillomviren / Chronisch degenerative Erkrankungen des zentralen Nervensystems / Retroviren / Picornaviren / Berichte der Referenzzentren.

Auskunft: Prof. Dr. med. F. Deinhardt, Max von Pettenkofer-Institut f. Hygiene u. Med. Mikrobiologie der Universität München, Pettenkoferstr. 9a, 8000 München 2, Tel.: 089/5160-5203.

Rom (Italien): 25 . bis 28 . Oktober 1988 - $20^{\circ}$ Congresso della Societa Italiana di Biochimica Clinica.

Auskunft:Segreteria Organizzativa, Congresso SIBioC'88, Emmezeta s.r.l., Via Carlo Farini 70, 1-20359 Milano MI.
Esslingen: 26. Oktober 1988 - Behandlung von Abfällen aus dem medizinischen Bereich.

Themen: Umsetzung der hygienischen Vorgaben zur Abfallerfassung / Abfallbehandlung im Krankenhaus / Praktische Aspekte der Abfallerfassung am Anfallort / Vermeidung/Verwertung von Abfällen aus dem medizi nischen Bereich / Abfallentsorgung aus dem medizinischen Bereich.

Auskunft: Technische Akademie Esslingen, Weiterbildungszentrum, Postfach 1269, 7302 Ostfildern.

Tübingen: 3. bis 4. November 1988 - Gaschromatographisch-massenspektrometrische Methoden in der biochemischen, klinisch-chemischen und toxikologischen Analytik.

Auskunft: Arbeitsstelle Wissensch. Fort- u. Weiterbildung, Wilhelmstr. 5 7400 Tübingen, Tel.: $07071 / 296439$.

Singapur (Singapur): 6. bis 11. November 1988 - 1st Asia-Pacific Congress of Medical Virology.

Auskunft: Dr. M. Doraisingham, Virology Section, Dept. of Pathology, Outram RD., Singapore 0316, Republic of Singapore.

Innsbruck (Österreich): 7. bis 12. November 1988 - Hämatologieund Immunologiekurs II für Fortgeschrittene.

Auskunft: Univ.-Klinik f. Innere Medizin, Kanzlei, Anichstr. 35, A-6020 Innsbruck, Tel.: 0043/5222/723/3390.

Wien (Österreich): 8. bis 11. November 1988 - International Meeting on Two-Dimensional Electrophoresis.

Themen:Forum for the newest technical aspects, applications and research results in the field of high resolution two-dimensional electrophoresis. (The high resolution 2-DE is a powerful technique for the development of new diagnostic procedures in medicine and in basic research e.g. in biochemistry and gentechnology.) Exhibition of instruments, reagents and literature.

Auskunft: Secretary General, Dr. A. T. Endler, c/o INTERCONVENTION, Austria Center Vienna, A-1450 Vienna, Tel.: 43-222-2369/647.

Graz (Österreich): 17. bis 19. November 1988 - 22. Jahrestagung der Österreichischen Gesellschaft für Tropenmedizin und Parasitologie.

Auskunft: Österreichische Gesellschaft für Tropenmedizin und Parasitologie, Kinderspitalgasse 15, A-1095 Wien.

Ulm: 23. bis 26 . November 1988 - Seminar über „Sepsis und septischer Schock"

Themen: Praktisch-klinische Gesichtspunkte.

Auskunft:Sekr. Prof. H. G. Berger, Chirurgische Universitäts-Klinik, Steinhövelstr. 9a, $7900 \mathrm{Ulm}$, Tel.: 0731/1792200.

Mailand (Italien): 24. bis 26. November 1988 - Advanced Technology for the Clinical Laboratory and Biotechnology. 4th European Edition of the Oak Ridge Conference.

Auskuntt: ATB '88, Via Farini, 70, 1-20159 Milano.

Essen: 24. bis 26. November 1988 - Spezialkurs zum Erwerb der Fachkunde im Strahlenschutz als ermächtigter Arzt - Teil II nach der Strahlenschutz-Gesetzgebung.

Auskunft: Haus der Technik e.V., Hollestr. 1, 4300 Essen 1, Tel.: 0201 / 1803-1.

Essen: 25. bis 26. November 1988 - Laser-Strahlenschutzkurs für Mediziner, Naturwissenschaftler, Ingenieure und Techniker zum Erwerb der Fachkunde im Strahlenschutz bei der Anwendung von Laserstrahlen im medizinischen und nichtmedizinischen Bereich, nach den „Unfallverhütungsvorschriften Laserstrahlen" (VBG 93).

Auskunft: Haus der Technik e.V., Hollestr. 1, 4300 Essen 1, Tel.: $0201 /$ 1803-1.

Esslingen: 28. November bis 2. Dezember 1988 - Strahlenschutz beim Umgang mit radioaktiven Stoffen und Röntgenstrahlen. Allgemeiner Grundkurs zum Fachkundenachweis für Strahlenschutzbeauftragte. Anerkannt nach $\S 37$ Abs. 7 BetrVG und $\S 46$ Abs. 7 B Pers VG. Auskunft: Technische Akademie Esslingen, Weiterbildungszentrum, Postfach 1269, 7302 Ostfildern.

Essen: 1. bis 3. Dezember 1988 - Strahlenschutz-Grundkurs für Mediziner und Nichtmediziner (z. B. Medizinphysiker, Chemiker, Biologen, die im medizinischen Bereich tätig sind), gemäß der Röntgen- und Strahlenschutzverordnung über den Erwerb der Fachkunde im Strahlenschutz bei der Anwendung von Rötgenstrahlen, radioaktiven Stoffen und $\mathrm{Be}$ strahlungsanlagen im medizinischen Bereich, entsprechend den neuen Richtlinien zu der Röntgenverordnung vom 8.Januar 1987 und der „Richtlinie Strahlenschutz in der Medizin", Anlage A3, Ziffer 1.1 und Anlage A3. Ziffer 2.1. Gemeinsames Ministerialblatt Nr. 31, S.638, vom 10. Nov. 1979. Auskuntt: Haus der Technik e.V., Hollestr. 1, 4300 Essen 1. Tel.: 0201 / $1803-1$. 
Wien (Osterreich): 1. bis 3. Dezember 1988 - Jubiläumstagung der Osterreichischen Gesellschaft für Klinische Chemie gemeinsam mit den Deutschen und Schweizerischen Gesellschaften für Klinische Chemie.

Themen: Molekularbiologische Analytik in der Klinischen Chemie / Diagnostische Strategie der extravasalen Köıperflüssigkeiten / Klinische Chemie und Pathobiochemie des Herzens / Neue pathobiochemische Aspekte und klinisch-chemische Parameter in der Leberdiagnostik, Tumordiagnostik. Schilddrüsendiagnostik und Hämostaseologie / Free Communica. tions.

Auskunft: Prof. Dr. Mathias M. Müller, II. Chirurgische Klinik, Klinische Biochemie, Spitalgasse 23, A-1090 Wien, Tel.: 0222/4800 - 2338.

Essen: 8 . bis 10. Dezember 1988 - Strahlenschutz-Kurs für medizinisch-technische Assistenten und Radiologie-Assistenten über den Erwerb der Kenntnisse im Strahlenschutz bei der Anwendung von Röntgenstrahlen am lebenden Menschen, gemäß der „Richtlinie Durchführung der Röntgenverordnung".

Auskunft: Haus der Technik e.V., Hollestr. 1, 4300 Essen 1, Tel.: $0201 /$ $1803-1$.

\section{Terminkalender}

\section{August 1988}

8. -12.8 .

22. -26.8 .

Kyoto: Intern. Magnesium Symposium (BDL 1988, 40) Pécs: Intern. Symp. on the Problems of Listeriosis of the Hungarian Society of Microbiology and the IUMS (BDL 1988, 40)

23.-26. 8. Ghent: Intern. Symp. on Mass. Spectrometry in Life Sciences (BDL 1988, 91)

25.-27. 8. Kaposvar: Annual Meeting of Hungarian Society of Microbiology (BDL 1988, 40)

28. -31.8 Karlsruhe: Therapiewoche Karlsruhe (BDL 1988, 40)

28. 8. - 2.9. Hongkong: Asian-Pacific Congress of Clinical Biochemistry (BDL 1987, 119)

28. 8. - 2. 9. Milano: Congress of the International Society of Haematology (BDL 1988, 79)

29. 8. - 2. 9. Tübingen: Humane Monoklonale Antikörper: Herstellung u. Anwendung (BDL 1988, 91)

\section{September 1988}

1.- 2. 9

Freiburg: Congress on Nosocomial Infection Control in Intensive Care (BDL 1988, 40)

3. - 5.9. Taipei:Intern. Symp. on "Human Tumor Markers" (Satellite Meeting to the 4th Asian-Pacific Congress of Clinical Biochemistry) (BDL 1988, 79)

5. - 8.9. Kobe: Intern. Congress on Automation and New Technology in the Clinical Laboratory (BDL 1987, 119)

5.- 9.9. Torun: Conference on Analytical Atomic Spectroscopy (CANAS) (BDL 1988, 40)

5.- 9. 9. Espoo: Intern. Conf. on Nickel Metabolism and Toxicology (BDL 1988, 40)

7.-10.9. Stuttgart: Medizin-Technik 88 (BDL 1988, 79)

8. -10.9 .

9. -12.9 .

Baden: MYK 88 (BDL 1988, 40)

Davos: Interdisziplinäre Forschung von AIDS (BDL 1988, 40)

10. - 14. 9. Sarajevo: Meeting of European Society for Haemapheresis (BDL 1988, 79)

11.-14.9. Osaka: Intern. Congress on Clinical Enzymology (BDL 1988, 40)

11.-17.9. Den Haag: Intern. Leprosy Congress (BDL 1988, 41)

12. - 14. 9. Innsbruck: Gemeins. Herbsttagung der Dt. Ges. f. Biologische Chemie u.d. Österreich. Biologischen Gesellsch. (BDL 1988, 41)

13.-15.9. Basel: Wissenschaftl. Tagung d. Ges. f. Versuchstierkunde (BDL 1988, 79)

14. -18. 9. Zürich: Symp. d. Dt. Ges. f. Transfusionsmedizin u. Immunhämatologie (BDL 1988, 79)

17. - 20.9. Freiburg: Vers. d. Gesellsch. Dt. Naturforscher ù. Ärzte (BDL 1988, 41)

18.-23. 9. Wien: Intern. Symp. on "Gas Chromatography” (BDL 1988, 79)

18.-23.9. Amsterdam: Intern. Congress on „Tropical Medicine and Malaria" (BDL 1988, 79)

19.-20.9. L'Aquila: Advances in Biotechnology of Membrane Ion Transport (BDL 1988, 41)

19. - 20. 9. Madrid: European Group for Rapid Viral Diagnosis (BDL 1988, 41)

19. 9.-14. 10. Berlin: Strahlenschutzkurs für Ärzte (BDL 1988, 79)

20.-23. 9. Stockholm: Intern. Conference on Human Tumor Markers (BDL 1988, 41)
21. -23.9 .

Tübingen: Praktische UV-NIS-Spektroskopie I (Grundlagenkurs) (BDL 1988, 41)

23.-24. 9. Stuttgart: Hohenheimer Magnesium-Symposium (BDL 1988, 41)

25. - 29. 9. Florenz: Intern. Symp. on Bioluminescence and Chemiluminescence (BDL 1988, 41)

25. - 29. 9. Wien: Intern. Symp. on Anaerobic Bacteria (BDL 1988 41)

26. - 30.9. Tübingen: Praktische UV-/VIS-Spektroskopie II (Fortgeschrittenenkurs) (BDL 1988, 41)

26. - 30.9. Tübingen: Grundkurs im Strahlenschutz (BDL 1988, 41)

27. 9.

28. -30.9 . San Diego: Intern. Symp. on Bioluminescence and Chemiluminescence (BDL 1988, 79)

Kiel: Arbeitstagung der Sektion III - Hygiene - u Sektion II - Mikrobiologie - der DGHM (BDL 1988, 79)

29. 9. - 1. 10. Essen: Strahlenschutz-Grundkurs f. Mediziner u. Nichtmediziner (BDL 1988, 91)

\section{Oktober 1988}

2. - 5. 10. Salzburg: Jahrestagung d. Österr. u. Dt. Ges. f. Hämatologie und Onkologie (BDL 1988, 80)

3. - 5. 10. Düsseldorf: Jahrestagung d. Arbeitsgemeinschaft $f$. Gendiagnostik e.V. (BDL 1988, 80)

3. - 7. 10. Rom: Intern. Symp. on „Epstein-Barr Virus and Associated Malignant Diseases" (BDL 1988, 91)

6. - 7. 10. Mainz: Schliersee-Symp.d. Fachgruppe med. Chemie d. Ges. Dt. Chemiker (BDL 1988, 80)

6. - 8. 10. Düsseldorf: XX. Tagung d. Gesellschaft für Immunologie (BDL 1988, 80)

6. - 8. 10. Essen: Spezialkurs zum Erwerb der Fachkunde im Strah lenschutz als ermächtigter Arzt - Teil I (BDL 1988, 91)

10. - 14. 10. Paris: European School of Haematology (BDL 1988, 80)

13. -15. 10. Essen: Strahlenschutz-Kurs f. med.-techn. Assistenten u. Radiologie-Assistenten (BDL 1988, 91)

13. -15.10 . Essen: Strahlenschutz-Spezialkurs f. Nuklearmediziner u. Labormediziner (BDL 1988, 91)

17. - 19. 10. Dresden: 6th Dresden Lipid Symposium (BDL 1987, 84) 21. -22.10 . Erlangen: Arbeitstagung der Dt. Vereinigung zur Bekämpfung der Viruskrankheiten e.V. (DVV): Molekularbiologie in der virologischen Diagnostik (BDL 1988, 91)

24. -28. 10. Jena: Immunologischer Weiterbildungslehrgang (BDL 1988, 80)

25. -28. 10. Rom: Congresso della Societa Italiana di Biochimica Clinica (BDL 1988, 91)

26. 10. Esslingen: Behandlung von Abfällen aus dem medizinischen Bereich (BDL 1988, 91)

\section{November 1988}

3.- 4. 11. Tübingen: Gaschromatographisch-massenspektroskopische Methoden in der biochem., klin.-chem. u. toxikol. Analytik (BDL 1988, 91)

6. - 11. 11. Singapur: 1st Asia-Pacific Congress of Medical Virology (BDL 1988, 91)

7. - 12. 11. Innsbruck: Hämatologie- u. Immunologiekurs II f. Fortgeschrittene (BDL 1988, 91)

8.-11. 11. Wién: Intern. Meeting on Two-Dimensional Electrophoresis (BDL 1988, 91)

10.-11. 11. Berlin: INSTAND-Symposium ,'Trends in der Hämatologie" (BDL 1988, 80)

17.-19. 11. Graz: Jahrestagung d. Öșterr. Ges. f. Tropenmedizin u. Parásitologie (BDL 1988, 91)

23.-26. 11. . Ulm: Seminar über „Sepsis und septischer Schock” (BDL 1988, 91)

24. - 26. 11. Mailand: Advanced Techn. for the Clin. Lab. and Biotechnology, 4th Europ. Edition of the Oak Ridge Conference (BDL 1988, 91)

24. - 26. 11. Essen: Spezialkurs zum Erwerb der Fachkunde im Strahlenschutz als ermächtigter Arzt - Teil II (BDL 1988, 91) 25.-26. 11. Essen: Laser-Strahlenschutzkurs (BDL 1988, 91)

28. 11. - 2. Esslingen: Strahlenschutz beim Umgang mit radioakti12. ven Stoffẹn und Röntgenstrahlen (BDL 1988, 91)

30. 11.-2. 12. Dresden: Kongreß d. Ges. f. Klin. Chemie u. Laboratoriumsdiagnostik der DDR (BDL: 1988, 62)

\section{Dezếmber 1988}

1.- 3. 12.

1. - 3. 12 .

Essen: Strahlenschutz-Grundkurs (BDL 1988, 91)

Wien: Jubiläumstagung der Österr. Ges. f. Klin. Chemie (BDL 1988, 92)

8. - 10. 12. Essen: Strahlenschutz-Kurs f. med.-techn. Assistenten u. Radiologie-Assistenten (BDL 1988, 91)

9. 12: Tübingen: Behandlung radioaktiver Abfälle in Mediẹin u. Forschung (BDL 1988, 80) 